\title{
Three-Dimensional, Space-Dependent Mesoscale Diffusivity: Derivation and Implications
}

\author{
V. M. CANUTO \\ NASA Goddard Institute for Space Studies, and Department of Applied Physics and Mathematics, Columbia University, \\ New York, New York \\ Y. CHENG \\ NASA Goddard Institute for Space Studies, and Center for Climate Systems Research, Columbia University, New York, New York

\section{A. M. HOWARD} \\ NASA Goddard Institute for Space Studies, and Department of Physics and Computer Science, Medgar Evers College of the \\ City University of New York, New York, New York

\section{S. DubOVIKov} \\ NASA Goddard Institute for Space Studies, and Center for Climate Systems Research, Columbia University, New York, New York
}

(Manuscript received 15 June 2018, in final form 30 January 2019)

\begin{abstract}
Recently, we presented a parameterization of an arbitrary tracer 3D mesoscale flux that describes both diabatic and adiabatic regimes without using arbitrary tapering functions. However, we did not parameterize the mesoscale diffusivity, which is the subject of this work. A key difference between the present and previous diffusivity parameterizations is that in the latter, the two main ingredients, mesoscale drift velocity and eddy kinetic energy, were not parameterized but determined using present data, which deprives the models of predictive power. Since winds, stratification, etc., are predicted to change in the future, use of these parameterizations to study future climate scenarios becomes questionable. In this work, we parameterize drift velocity and eddy kinetic energy (vertical-horizontal components), which we first assess with data [WOCE, TOPEX/Poseidon (T/P), and North Atlantic Tracer Release Experiment (NATRE)] and then use in a coarse-resolution stand-alone ocean code under Coordinated Ocean-Ice Reference Experiment I (CORE-I) forcing. We present results for the global ocean temperature and salinity, Atlantic overturning circulation, meridional heat transport, and Drake Passage transport, which we compare with several previous studies. The temperature drift is less than that of five of seven previous OGCMs, and the salinity drift is among the smallest in those studies. The predicted winter Antarctic Circumpolar Current mixed layer depths (MLDs) are in good agreement with the data. Predicting the correct MLD is important in climate studies since models that predict very deep mixed layers transfer more of the radiative perturbation to the deep ocean, reducing surface warming (and vice versa).
\end{abstract}

\section{Mesoscale parameterizations}

Coarse-resolution ocean global circulation models (OGCMs) cannot resolve mesoscales $(10-100 \mathrm{~km}$, lifetime on the order of months; e.g., Frenger et al. 2015), which must be parameterized. The OGCM equation for an arbitrary mean tracer $\bar{c}$ (temperature $T$, salinity $S$, concentration) is given by

$$
\partial_{\mathrm{t}} \bar{c}+\overline{\mathbf{U}} \cdot \nabla \bar{c}+\nabla \cdot \mathbf{F}(\bar{c})=\text { sources }- \text { sinks, }
$$

\footnotetext{
Corresponding author: V. M. Canuto, vmcanuto@gmail.com
}

where $\overline{\mathbf{U}}$ is the $3 \mathrm{D}$ mean velocity and $\mathbf{F}(\bar{c})$ is the $3 \mathrm{D}$ mesoscale-induced tracer flux:

$$
F_{i}(\bar{c})=-\kappa_{M} K_{i j} \partial_{j} \bar{c},
$$

where $\kappa_{M}$ is the mesoscale diffusivity and $K_{i j}$ is the dimensionless 3D diffusivity tensor whose form was presented in Canuto et al. [2018, hereafter C18, their relations (7.5) and (7.6)]; it differs from the $K_{i j}$ employed thus far since it includes mesoscale features not accounted heretofore. Specifically, C18 includes two 
different regimes, the deep adiabatic (A) regime and the upper-layers' diabatic (D) regime, that are physically different and governed by different conservation laws. $\mathrm{C} 18$ also includes a key finding from TOPEX/Poseidon (T/P) altimetry data (Chelton et al. 2011, hereafter C11), namely, that mesoscales do not travel with the mean velocity but with their own translational or drift velocity that was absent in previous parameterizations. Though in $\mathrm{C} 18$ we described the A-D regimes, it is useful to enlarge that description, which we do in appendix A. The C18 parameterization did not provide a model for $\kappa_{M}$, which is the goal of this work. The new parameterization is then used in a coarse-resolution OGCM discussed in section 6 and the results are presented in section 7 .

\section{Mesoscale diffusivity $\kappa_{M}$}

The derivation of a mesoscale diffusivity presents several aspects. A first issue is how to assess the result with the data. In a recent work, Roach et al. (2018) presented an instructive discussion of this issue, specifically Argo and near-surface drifter data, and their Fig. 8 is particularly useful in that respect. The second item concerns C11's conclusion that "essentially all of the observed mesoscale features are non-linear." This implies that a treatment of $\kappa_{M}$ based on linear analysis is not appropriate, and yet several models for $\kappa_{M}$ (e.g., Bates et al. 2014; Roach et al. 2018) employ ingredients from the linear theory with the following consequences. Two key ingredients of the diffusivity are the mesoscale phase velocity and eddy kinetic energy (EKE), neither of which can be computed with a linear model. Bates et al. (2014) and Roach et al. (2018) used present data by Lebedev et al. (2007) and $\mathrm{Fu}$ (2009), a procedure that calls into question the applicability of the results to future climate scenarios when conditions different than today may significantly alter wind stress, EKE, and phase speed. In this work, we employ the nonlinear mesoscale model developed in Canuto and Dubovikov (2005, hereafter CD5) and Canuto and Dubovikov (2006, hereafter CD6) that yields (A.2) for the drift velocity $\mathbf{u}_{d}$ that $\mathrm{C} 11$ considered the "most germane" of all the nonlinear metrics; in Fig. 1 of $\mathrm{C} 18$ we assessed such an expression against $\mathrm{T} / \mathrm{P}$ data. EKE is computed in sections 3 and 4 using the same nonlinear model and which we assess using WOCE and T/P data. Specifically, we solve the stationary, local limit of the EKE equation, which requires the form of the energy source, and since analysis of altimetry data concluded that "nearly all of the world ocean is baroclinically unstable" (C11, their section 3.4), one needs an expression for the vertical mesoscale buoyancy flux for both adiabatic and diabatic
(A-D) regimes that were given in section 3 of C18. As in the case of the drift velocity, the D-regime buoyancy flux was assessed using a mesoscale-resolving numerical simulation (Luneva et al. 2015). In summary, the CD5 nonlinear mesoscale model used in this work provides the expressions of the eddy drift velocity and eddy kinetic energy $K$. What remains to be discussed is the mesoscale diffusivity. The problem has two parts, the first of which is the use of the mixing length theory (MLT; Prandtl 1925) represented by the relation $\left(r_{d}\right.$ is the Rossby deformation radius):

$$
\kappa_{M}=r_{d} K^{1 / 2} .
$$

\section{a. Does the MLT (2.1) overestimate the diffusivity?}

Relation (2.1) is based on the assumption that there is only one "mixing length" represented by $r_{d}$. However, since a turbulent regime is characterized by a wide range of eddies of different sizes, the assumption of "one mixing length only " must be examined. The kinetic energy spectrum $E(k)$, whose integral over all wavenumbers $k\left(k \propto \ell^{-1}, \ell\right.$ being the size of the eddy) yields the eddy kinetic energy $K$, describes all the eddies from the largest to the smallest one where the kinematic vis$\operatorname{cosity} \nu$ leads to dissipation. The width of the spectrum $E(k)$ depends on $\nu$, the larger the $\nu$, the narrower is the width of $E(k)$ and vice versa. Specifically, the ratio of the largest to the smallest eddy scales like $\mathrm{Re}^{3 / 4} \propto \nu^{-3 / 4}$, where $\mathrm{Re}$ is the Reynolds number (e.g., McComb 1992). The form of the turbulent viscosity felt by an eddy of arbitrary size $k^{-1}$ was derived in Canuto and Dubovikov [1996, their (24)] to have the form

$$
\nu_{t}(k)=\left[\nu^{2}+\frac{1}{2} \int_{k}^{\infty} q^{-2} E(q) d q\right]^{1 / 2}-\nu,
$$

where the sum $\nu+\nu_{t}(k)=\nu_{d}(k)$ is often called dynamical viscosity. A good feature of (2.2) is that it describes both small and large eddies: 1) for small eddies (large $k$ ), the integral is small and the turbulent viscosity is small, and 2) for large eddies (small $k$ ), the integral is large, the kinematic viscosity is negligible, and the turbulent viscosity is large. If one knows the eddy spectrum $E(k)$, one can account for the contribution of all the eddies to the turbulent viscosity. Denoting $k_{0} \propto r_{d}^{-1}$ the largest eddy, the lower limit in the integral (2.2) becomes $k_{0}$ and the integration over increasingly large wavenumbers then accounts for the contribution of all eddies smaller than $r_{d}$. Let us rewrite (2.2) in a form that exhibits the MLT model (2.1). Using $\kappa_{M}\left(k_{0}\right)=\sigma_{t}^{-1} \nu_{t}\left(k_{0}\right)$, where $\sigma_{t}=0.72$ is the turbulent Prandtl number, neglecting the kinematic viscosity, (2.2) gives 


$$
\kappa_{M}=\alpha r_{d} K^{1 / 2}, \alpha^{2} \equiv \frac{k_{0}^{2}}{2 \sigma_{t}} \frac{\int_{k_{0}}^{\infty} q^{-2} E(q) d q}{\int_{k_{0}}^{\infty} E(q) d q},
$$

where we used the relation $k_{0} r_{d}=\sigma_{t}^{-1 / 2}$ that was derived in CD5 [their (13b)]. If we consider a power spectrum of the type $E(q)=E_{*} q^{-m}$, we have

$$
\alpha=\left(\frac{1}{2 \sigma_{t}} \frac{m-1}{m+1}\right)^{1 / 2} \simeq \frac{1}{2},
$$

where we used $\sigma_{t}=0.72$ and $m=3$ corresponding to geostrophic turbulence (Charney 1971; Scott and Wang 2005). This shows that the MLT (2.1) with $\alpha=1$ overestimates the mesoscale diffusivity and the factor $1 / 2$ may help explain the result of Roach et al. (2018, their section 5) that the "estimates are 1.5-2 times greater than the observed diffusivities." The assumption $E(k)=E_{*} k^{-m}$ can be improved on as follows. The procedure requires solving the equation for $E(k)$ provided by turbulence models, which depends on the form of the forcing mechanism, which in the ocean is primarily baroclinic instabilities. Given the ample literature on baroclinic instabilities (e.g., Stone 1966) the form of the growth rates needed to solve the $E(k)$ equation is known and the above procedure can be pursued. We can cite two examples of such an approach, turbulent channel flow and thermal convection (Canuto et al. 1990; Canuto and Mazzitelli 1991). In the second case, the form of $E(k)$ was solved for three values of the kinematic Prandtl numbers (ratio of kinematic viscosity to thermal conductivity) and it exhibited the expected behavior: the larger the kinematic viscosity, the narrower the spectrum.

\section{b. Mean flow-mesoscales interaction}

We employ the CD5 nonlinear mesoscale model to derive (appendix B) how the effect of the mesoscalemean flow interaction changes $[(2.3)]$ :

$$
\kappa_{M}=\alpha r_{d} K^{1 / 2} \varpi\left(\mathbf{u}_{D}, K\right),
$$

where

$$
\varpi=\left(1+\frac{1}{K}\left|\mathbf{u}_{D}\right|^{2}\right)^{-1 / 2}
$$

where $\mathbf{u}_{D} \equiv \overline{\mathbf{u}}-\mathbf{u}_{d}$ and $\overline{\mathbf{u}}$ is the mean velocity. A few comments are necessary. First, because of Galilean invariance, drift and mean velocities appear as the relative velocity $\mathbf{u}_{D}$. Second, to the main order in $K^{-1}\left|\mathbf{u}_{D}\right|^{2}$, relation (2.6) becomes

$$
\varpi \cong\left(1+\frac{\left|\mathbf{u}_{D}\right|^{2}}{2 K}\right)^{-1},
$$

which is closer to the form used in serval recent papers, as we discuss below. Third, in the dynamic equations of the mesoscale velocity, the tendency term yields $\partial_{t} \mathbf{u} \rightarrow$ $i \omega \mathbf{u}$ and the advection term yields $\mathbf{u} \cdot \nabla \mathbf{u} \rightarrow(\mathbf{k} \cdot \mathbf{u}) \mathbf{u}$. As discussed in detail in CD5, after the mesoscale equations are transformed into an eigenvalue problem for the Bernoulli function, the dispersion relation was found to be $\omega=\mathbf{k} \cdot \mathbf{u}_{d}$, which yields the form of $\mathbf{u}_{d}$ [(A.2)]. Tendency and advection combine to yield the term $\overline{\mathbf{u}}-\mathbf{u}_{d}$ in (2.6) that tends to "suppress" the diffusivity.

\section{c. Previous forms of the suppression factor}

To quantify this factor, Meredith et al. (2012), Bates et al. (2014), Klocker and Abernathey (2014), Roach et al. (2018), and Busecke and Abernathy (2019, hereafter BA) employed results from stability analysis (Ferrari and Nikurashin 2010, hereafter FN). The relation of stability analysis with fully developed turbulence was discussed by Lumley and Yaglom (2001, their section 2.0.4), who credited Corrsin and Liepmann for the idea. Corrsin and Liepmann concluded that "stability theory had nothing to do with turbulence." The FN result was translated into the form (Bates et al. 2014)

$$
\varpi(\bar{u}-c, K)=\left(1+b_{1} \frac{|\bar{u}-c|^{2}}{u_{\mathrm{rms}}^{2}}\right)^{-1},
$$

where $b_{1}$ is an adjustable parameter, $\bar{u}$ is the mean velocity, and $c$ is the eddy phase speed. The eddy kinetic energy $u_{\mathrm{rms}}^{2} / 2$ was not parameterized but computed using altimetry data, and the phase velocity $c$ was also not parameterized but computed using altimetry data (Smith and Marshall 2009, their footnote 1). Figure 10a of Bates et al. (2014) compares (2.8) with North Atlantic Tracer Release Experiment (NATRE) data: above $0.5 \mathrm{~km}$, the best fit is with $b_{1}=0$, which implies no mesoscale-mean flow interaction while below $0.5 \mathrm{~km}$, neither of the suggested values $b_{1}=1,4$ can reproduce the data. Since two of the ingredients in (2.8) were taken from present data, the relation lacks predictive power. Recently, BA wrote the mesoscale diffusivity in the following form:

$$
\kappa_{M}=c r_{d} K^{1 / 2} \varpi\left(\mathbf{u}_{D}, K\right), \quad \varpi=\left[1+\alpha\left(c_{w}-U\right)^{2}\right]^{-1},
$$

where the coefficient $c=0.5$ and $U$ is the zonal mean flow. If we compare these relations with (2.5)-(2.7), we reach the following conclusions. In BA the phase speed is taken to be ( $U^{z t}$ is the depth- and time-averaged zonal flow)

$$
c_{w}=U^{z t}-\beta r_{d}^{2},
$$


whereas in the present model it is given by relation (A.2):

$$
\mathbf{u}_{d}(x, y)=\langle\overline{\mathbf{u}}\rangle+\sigma \mathbf{c}_{R}+\text { nonlinear terms } .
$$

In (2.10) the mean velocity $U$ is vertically averaged while in $(2.11)\langle\ldots\rangle$ is a vertical integration with a weight $\kappa_{M}$,

$$
\langle\ldots\rangle=\frac{\int \ldots \kappa_{M}(z) d z}{\int \kappa_{M}(z) d z},
$$

which ensures the known fact that the diffusivity is surface enhanced and equally important depends on stratification. In (2.6) and (2.7) the kinetic energy $K$ has vertical variation shown in Fig. 1 exhibiting the surface enhancement, and surface values that are strongly location dependent, as shown by the altimetry $\mathrm{T} / \mathrm{P}$ data (presented later in Fig. 8). On the other hand, in the second part of (2.9), $K$ is represented by the quantity $\alpha$, which BA assumed to be constant. This means that $\varpi$ is determined only by the mean velocity. BA's main conclusion that "changes driven by variations in the large-scale flow often exceed the effect of the eddy kinetic energy $K$ as a primary driver of variability of eddy mixing," is therefore predicated on the assumption that there is no $K$ dependence in $\varpi$. Such a dependence, once accounted for as in the present model [(2.6) and (2.7)], is likely to alter the above conclusion since $K$ enters in a way that mitigates the variations in the mean velocity. It would therefore be interesting to employ relations (2.5)-(2.7) to assess to what extent they alter the main conclusions by BA.

\section{Eddy kinetic energy: Vertical profile}

The three-dimensional nature of the mesoscale diffusivity (2.5) is due to the eddy kinetic energy that has a vertical profile $\Gamma(x, y, z)$ and a surface value $K_{s}(x, y)$ :

$$
K(x, y, z)=\Gamma(x, y, z) K_{s}(x, y),
$$

both of which are location dependent. In CD6 [their Eq. (5b)], the $z$ profile was derived to have the following form:

$$
\Gamma(x, y, z)=\left|1+a_{0}\right|^{-2}\left|a_{0}+B_{1}(z)\right|^{2} .
$$

Here, $B_{1}(z)$ is the first baroclinic mode solution of the eigenvalue problem $\partial_{z z} \varphi+\left(N / f r_{d}\right)^{2} \varphi=0, \varphi=$ $N^{-2} \partial_{z} B_{1}$ with the boundary conditions $\partial_{z} B_{1}=0$ at $z=-H_{b}, 0$ and $B_{1}(0)=1$, where $H_{b}$ is the depth of the ocean. Relation (3.2) represents a partition between the baroclinic component $B_{1}(z)$ and the barotropic component $a_{0}$. Wunsch (1997) concluded that the first
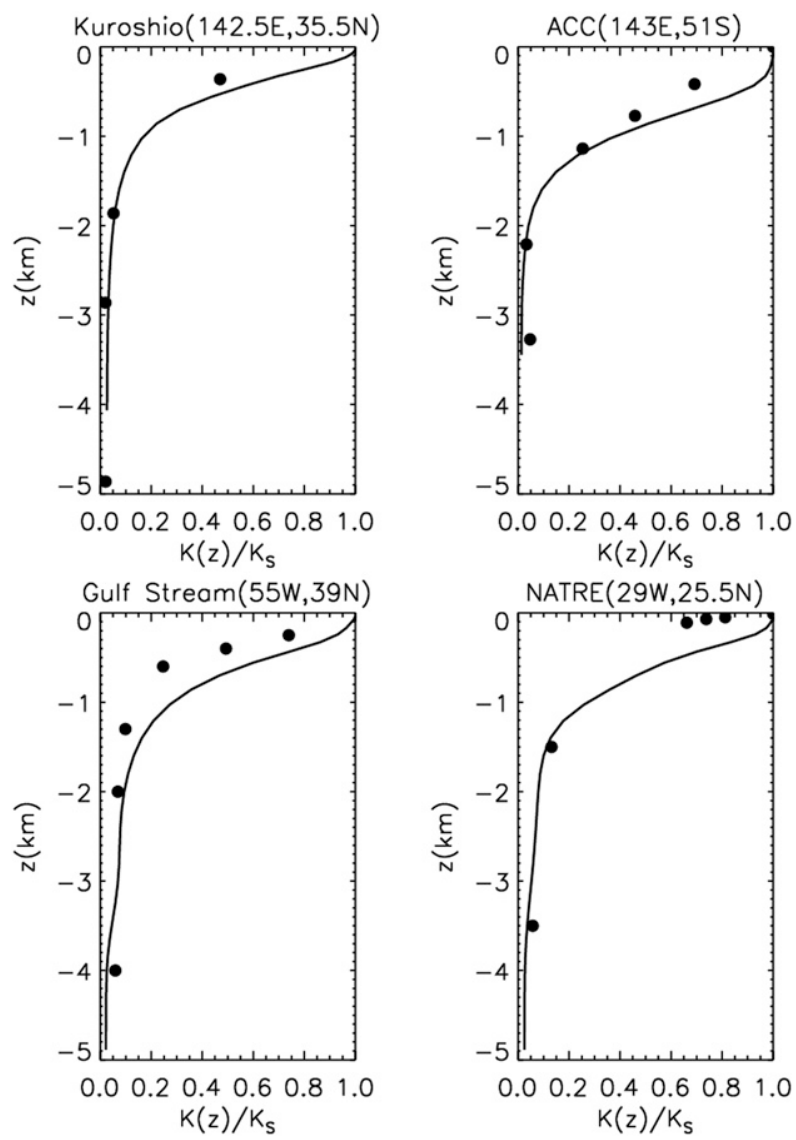

FIG. 1. Vertical profiles of the eddy kinetic energy equation (3.2) (solid line) vs WOCE (2002) data (black dots). The form of the barotropic contribution $a_{0}$ is the same in all four locations, but the specific value depends on the depth of the ocean at that location. The results are from the GISS-ER OGCM that is described in section 6. The OGCM data are averages over the last 3 years of a 500-yr simulation in this figure and in succeeding ones unless stated otherwise.

baroclinic mode $B_{1}(z)$ dominates near the surface. The form of $a_{0}^{2}$ was also derived in CD5 [their (24b), (24c)] but required an iterative process that is time consuming in an OGCM; after trials and errors, we concluded that $a_{0}^{2}=\left|B_{1}\left(-H_{b}\right)\right|$ was a good representation of the CD5 relation, and it is the one we use in this work. Figure 1 shows the $z$ profile (3.2) compared to WOCE (2002) data in different locations. ${ }^{1}$ Model (3.2) reproduces the data satisfactorily.

\footnotetext{
${ }^{1}$ The WOCE (2002) website does not provide EKE but time series of velocity profiles. We separated the latter into a mean (denoted by an overbar) and a fluctuating part (denoted by a prime $):(u, v)=(\bar{u}, \bar{v})+\left(u^{\prime}, v^{\prime}\right)$, where $(u, v)$ are the $(x, y)$ components of the velocity from WOCE and the overbar denotes an average over 3 months. The EKE was then obtained using $2 \mathrm{EKE}=\overline{u^{\prime 2}}+\overline{v^{\prime 2}}$.
} 
To put relation (3.2) in the proper context, we recall that in the absence of a model for the vertical profile of the eddy kinetic energy, authors suggested heuristic relations with the purpose of endowing the mesoscale diffusivity with an enhanced surface behavior. The need to do so had been advocated by several authors, and Farneti et al. (2015) have recently pointed out that failure to implement a 3D form of $\kappa_{M}$ resulted in major drawbacks, particularly in the representation of the Southern Ocean responses to climate change, heat uptake, eddy compensation, and carbon sequestration. A common suggestion was to assume that the mesoscale diffusivity scales like $N^{2}$ where $N$ is the Brunt-Väisälä frequency (Ferreira et al. 2005; Danabasoglu and Marshall 2007; Hofman and Morales Maqueda 2011; Farneti and Gent 2011; Gent and Danabasoglu 2011; Kuhlbrodt et al. 2012; Bryan et al. 2014; Gent 2011, 2016; Poulsen et al. 2018). Since (3.2) exhibits a surface enhancement, heuristic relations are no longer necessary. A final point worth mentioning is that the heuristic relations were not assessed before their use in OGCMs, whereas relation (3.2) is assessed using WOCE data (see Fig. 1). The surface eddy kinetic energy is discussed next.

\section{Eddy surface kinetic energy $K_{s}$}

Since no parameterization of $K_{s}$ is available in the literature (the only data are from numerical simulations and direct measurements), it was tempting to follow previous authors who employed $\mathrm{T} / \mathrm{P}$ altimetry data to determine $K_{s}$. The reason we do not follow this procedure is because the T/P data represent today's conditions but not necessarily future ones. For example, in the period 1980-2010 there was a $20 \%$ increase in the Antarctic Circumpolar Current (ACC) zonal wind stress that climate models predict will continue (e.g., Bi et al. 2002; Saenko et al. 2005; Boning et al. 2008), which will affect $K_{s}$.

\section{a. Baroclinic instabilities}

Since analysis of altimetry data concluded that "nearly all of the world ocean is baroclinically unstable" (C11, their section 3.4; Gill et al. 1974; Robinson and McWilliams 1974; Stammer 1998; Smith 2007), we determine $K_{s}(x, y)$ as the solution of the volumeintegrated, stationary limit of the eddy kinetic energy equation, which becomes the energy production = dissipation balance:

$$
\underbrace{\int_{-H_{b}}^{-h_{*}} F_{v}(\bar{b}) d z}_{\text {A regime }}+\underbrace{\int_{-h_{*}}^{0} F_{v}(\bar{b}) d z}_{\text {D regime }}=\int_{-H_{b}}^{0} \varepsilon(z) d z,
$$

where the production term is given by the mesoscale vertical buoyancy flux $F_{v}(\bar{b})$ representing baroclinic instabilities, $h_{*}$ is the depth at which the A-D regimes match (see section 5), $H_{b}$ is the ocean depth, and $\varepsilon$ is the rate of dissipation of $K(x, y, z)$. The definition and features of the A-D regimes are discussed in appendix A and the A-D vertical buoyancy fluxes are given in $\mathrm{C} 18$ (their sections $1 \mathrm{a}$ and $1 \mathrm{~b}$ ) by the second relation in their (2.3), (3.4a), (3.4b), and (3.6). Since both A-D fluxes depend linearly on $\kappa_{M}$, we use (2.5), (3.1), and (3.2) to exhibit the dependence on $K_{s}$ of the production terms in (4.1):

$$
\int_{-H_{b}}^{-h_{*}} F_{v}(\bar{b}) d z=K_{s}^{1 / 2} K_{A}, \quad \int_{-h_{*}}^{0} F_{v}(\bar{b}) d z=K_{s}^{1 / 2} K_{D},
$$

where

$$
\begin{aligned}
& K_{A}=\alpha r_{d} \int_{-H_{b}}^{-h_{*}} \varpi \Gamma^{1 / 2}(z) N^{2} \mathbf{s} \cdot \boldsymbol{\xi} d z, \\
& K_{D}=\alpha r_{d} \int_{-h_{*}}^{0} \varpi \Gamma^{1 / 2}(z) N^{2} \mathbf{s} \cdot \boldsymbol{\Omega} d z,
\end{aligned}
$$

where the vectors $\boldsymbol{\xi}$ and $\boldsymbol{\Omega}$ are defined in (A.1), (A.4), and (A.5). Relations (4.2) show that the production terms in (4.1) scale like $K_{s}^{1 / 2}$.

\section{b. Dissipation}

As done by previous authors (Eden and Greatbatch 2008), we assume that this variable can be described using the Kolmogorov kinetic energy spectrum $E(k)=$ $\mathrm{Ko} \varepsilon^{2 / 3} k^{-5 / 3}$, where Ko is the Kolmogorov constant $5<$ Ko $<8$ (Danilov and Gurarie 2000). Integrating $E(k)$ over all wavenumbers from $k_{0}$, one obtains $2 K=$ $3 \mathrm{Ko} \varepsilon^{2 / 3} k_{0}^{-2 / 3}$. Using $k_{0}^{-1}=r_{d} \sigma_{t}^{1 / 2}$ [CD5, their (13b)], where the turbulent Prandtl number $\sigma_{t}$ was introduced before in (2.3), and (3.1), after simple transformations, the rate of dissipation is derived to be

$$
\begin{aligned}
\langle\varepsilon\rangle_{\mathrm{Ko}} & \equiv \int_{-H_{b}}^{0} \varepsilon(z) d z=\alpha_{K} K_{s}^{3 / 2}, \\
\alpha_{K} & \equiv\left(C_{k} r_{d}\right)^{-1} \int_{-H_{b}}^{0} \Gamma^{3 / 2}(z) d z, \\
C_{K} & \equiv\left(\frac{3}{2} K o\right)^{3 / 2} \sigma_{t}^{1 / 2},
\end{aligned}
$$

which exhibits a $K_{s}^{3 / 2}$ scaling. Placing (4.4), (4.2), and (4.3) into (4.1), the latter becomes a linear algebraic equation in $K_{s}$ whose solution exhibits a global pattern similar to that of the T/P data (Scharffenberg and Stammer 2010; see Fig. 8, lower panel), but the values 
were too large in the equatorial region and too small in the ACC. This is an instructive result that had not been pointed out in previous studies that employed Kolmogorov's law but which is not surprising if one considers that it relies on the eddy kinetic energy, which is surface enhanced (Fig. 1), while many studies have stressed the importance of bottom dissipation. We thus tried the formulation of Cessi (2008), whose relations (13) and (19) yield the following form of the bottom dissipation $\varepsilon_{\mathrm{BD}}$ :

$$
\varepsilon_{\mathrm{BD}}=K_{s} \Lambda, \quad \Lambda \equiv \frac{2}{\tau} \int_{-H_{b}}^{-h_{*}} d z \Gamma(z) \gamma(z),
$$

where $\gamma(z)=(2 / \pi)^{1 / 2}\left(H_{b} / \delta_{b}\right) \exp \left(-\xi^{2} / 2\right), \xi \equiv\left(z+H_{b}\right) \delta_{b}^{-1}$ with $\delta_{b}=40 \mathrm{~m}$; in Table 1 of Cessi (2008) the time scale $\tau=(2.5-5) \times 10^{6} \mathrm{~s}$ was assumed to be constant. Using relation (4.5) in (4.1) together with (4.2) and (4.3) yielded a $K_{s}$ map that was spotty and even farther from the T/P data than in the Kolmogorov case. However, since (4.5) was originally intended for use in a limited ocean region, it is not surprising that, once extended to the whole ocean, it exhibits spottiness. Since reasonable changes of $\delta_{b}$ would not eliminate the spottiness, we focused on the time scale $\tau$ and reasoned that while the values in Table 1 of Cessi (2008) exhibit the correct magnitude, they cannot be assumed to be valid everywhere, independently of location. Thus, we introduced the following new feature. We use the definition of $\tau$ as the ratio of the average kinetic energy to the average rate of dissipation:

$$
\tau=\frac{2\langle K\rangle}{\varepsilon}=2 C_{k} \frac{r_{d}}{K_{s}^{1 / 2}} \frac{\int_{-H_{b}}^{0} \Gamma(z) d z}{\int_{-H_{b}}^{0} \Gamma^{3 / 2}(z) d z} .
$$

The key new feature in (4.6) is that $\tau$ is no longer a constant as it now depends on the location dependent surface eddy kinetic energy. Using $r_{d}=10 \mathrm{~km}, K_{s}=$ $10^{-2} \mathrm{~m}^{2} \mathrm{~s}^{-2}$, we obtain $\tau \sim 2 \times 10^{6} \mathrm{~s}$, which is similar to the values in Cessi (2008). Repeating the previous procedure, we now obtain

$$
\begin{aligned}
\alpha_{K} K_{s} & =(1+\mathrm{BD})^{-1}\left(K_{A}+K_{D}\right), \\
\mathrm{BD} & \equiv\left[\int_{-H_{b}}^{0} \Gamma(z) d z\right]^{-1} \int_{-H_{b}}^{-h_{*}} \gamma(z) \Gamma(z) d z,
\end{aligned}
$$

where BD stands for bottom dissipation. We therefore suggest (4.7) instead of (4.5). In section 7 we compare (4.7) with T/P data.

\section{c. Shear contribution}

In the presence of strong currents, for example, the Kuroshio, Gulf Stream, and ACC, relation (4.1) is bound to underestimate $K_{s}$ since it only accounts for baroclinic instabilities while to account for the shear contribution, the energy balance equation (4.1) must be modified as follows:

$\int_{-H_{b}}^{0} d z F_{V}(\bar{b})+\int_{-H_{b}}^{0} d z P_{s}=\int_{-H_{b}}^{0} d z \varepsilon(z), \quad P_{s}=-\overline{u_{i}^{\prime} u_{j}^{\prime}} S_{i j}$

where $P_{s}$ is the source of kinetic energy due to the interaction of the mesoscale momentum fluxes (Reynolds stresses) $\overline{u_{i}^{\prime} u_{j}^{\prime}}$ with the mean shear $2 S_{i j}=\bar{u}_{i, j}+\bar{u}_{j, i}$ $\left(a_{i, j}=\partial a_{i} / \partial x_{j}\right)$. Regrettably, at present, no parameterization of the Reynolds stresses is available, and the data point to a rather complex situation. For example, Fig. 13 of von Storch et al. (2012) shows that the mean flow feeds mesoscales in the upper $2-3 \mathrm{~km}$ while below that, the opposite occurs. Bryden and Brady (1989) showed that in the equatorial Pacific, use of a downgradient model such as $\overline{u_{i}^{\prime} u_{j}^{\prime}}=-2 \nu_{t} S_{i j}$ implies that $\nu_{t}>0$ above the equatorial undercurrent and $\nu_{t}<0$ below it. To assess the role of $P_{s}$, we used data from Wilkin and Morrow (1994, their Plate 5) that show that at $40^{\circ} \mathrm{S}$ the surface value of $P_{s}$ is

$$
P_{s} \simeq 10^{-7} \mathrm{~m}^{2} \mathrm{~s}^{-3} .
$$

In this case the first relation in (4.7) becomes

$$
\alpha_{K} K_{s}^{3 / 2}=(1+\mathrm{BD})^{-1}\left(K_{A}+K_{D}\right) K_{s}^{1 / 2}+h_{*} P_{s},
$$

where the second integral on the left-hand side of (4.8) was approximated by $h_{*} P_{s}$. Using (4.9), the results of (4.10) are shown (see the red and blue dots in Fig. 10 below).

\section{Depth of the $D$ regime}

In the A regime, diapycnal fluxes are negligible and the flow is primarily along isopycnal surfaces. By contrast, in the upper layers' D regime, diapycnal fluxes are large and water parcels no longer move along isopycnal surfaces. In the literature, the mixed layer depth (MLD) is often used to define the extent of the D regime. Gregory (2000, their section 2) presented reasons for the inadequacy of the MLD to represent the near-isothermal upper layers: MLD is a variable in both space and time, there is only limited geographical similarity between the MLD, and the penetration of, say, temperature change and no well-defined isothermal layer is apparent in the global average temperature profile (see Fig. 1 of Gregory 2000). 

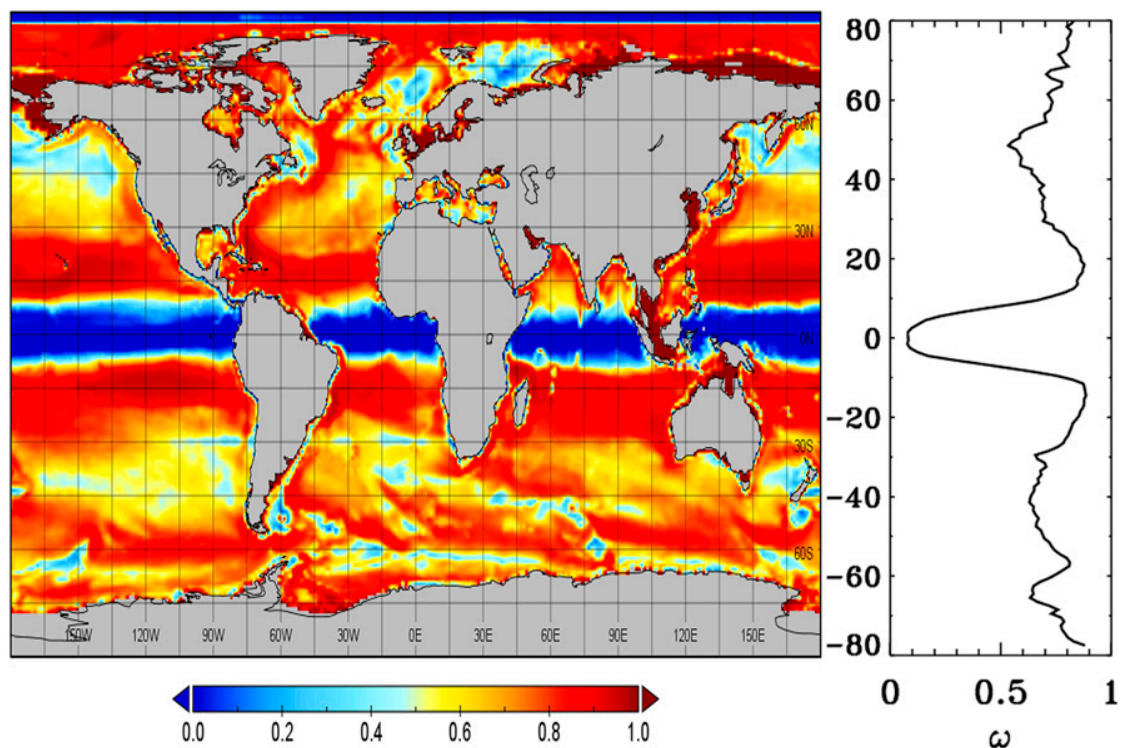

FIG. 2. Surface value of the function $\varpi[(2.6)]$ and its zonal average.

In the KPP vertical mixing scheme that we employ in this work (Large et al. 1994), the extent of the vertical mixing translates into a criterion to estimate the boundary layer depth (HBL) in terms of the bulk Richardson number $\mathrm{Ri}_{b}$. Essentially, HBL is a measure of how deep into a turbulent boundary layer an eddy with a near-surface velocity and buoyancy can penetrate into the interior stratification before becoming stable, in a Richardson number sense, relative to the local velocity and buoyancy. Numerically, it is the shallowest depth at which the bulk Richardson number exceeds a critical value estimated to be between 0.3 and 1 . Since reproducing the form of both $\mathrm{HBL}$ and $\mathrm{Ri}_{b}$ would require lengthy equations and the definition of several variables, we refer the reader to the original article by Large et al. (1994,

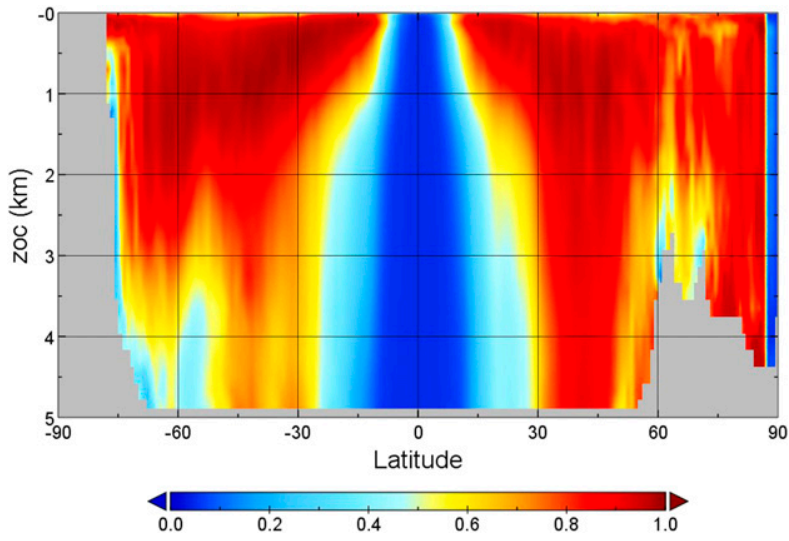

FIG. 3. Zonal average of the function $\varpi[(2.6)]$ at all depths.
371-372) and the summary by Large et al. (1997). Since the Gent-McWilliams (GM) model entails a relatively low level of stratification, there was no need for a strong vertical mixing and many studies chose the lowest value of 0.3 . The present mesoscale model actually produces a stronger stratification than GM as shown in C18 (their section 9 and Fig. 11) and thus we had to adopt a stronger vertical mixing, that is, a value larger than 0.3 . We employed the upper limit $\mathrm{Ri}_{b}(\mathrm{cr})=1$ which generally improves the results.

However, even the adoption of HBL in lieu of the MLD is still insufficient since it does not determine the

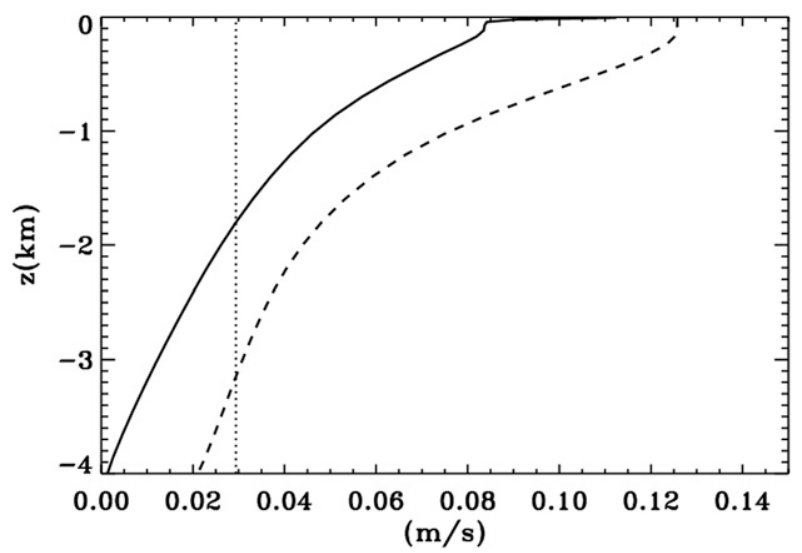

FIG. 4. Depth profiles averaged between $61^{\circ}$ and $56^{\circ} \mathrm{S}$ and between $110^{\circ}$ and $80^{\circ} \mathrm{W}$ of mean velocity (solid line), eddy drift velocity (dotted line), and square root of eddy kinetic energy (dashed line). 


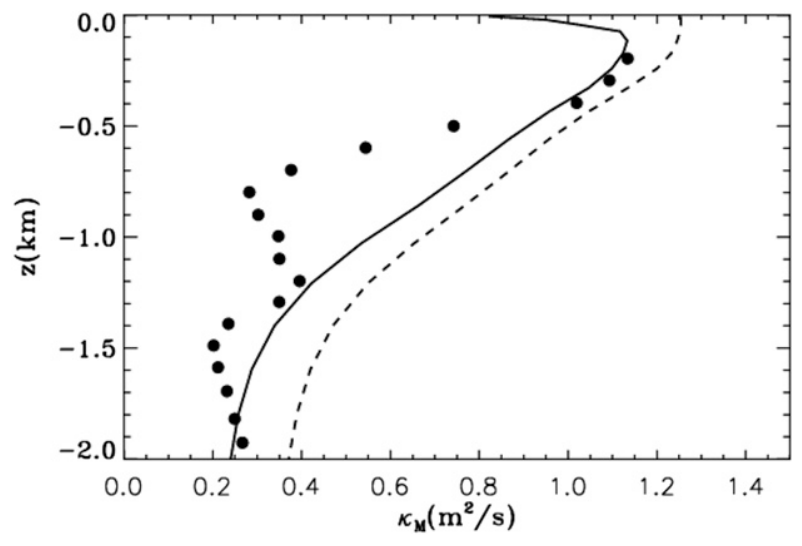

FIG. 5. Vertical profiles of the mesoscale diffusivity from (2.5)(2.6) (solid line) vs the data at NATRE (dots; Ferrari and Polzin 2005). The dashed line denotes the model result with the suppression factor $\varpi=1$. The data are from Fig. 10a of Bates et al. (2014).

depth $h_{*}$. Our suggestion is that $h_{*}$ should be less than the depth of the thermocline since at that depth the stratification is too strong for the $D$ regime to exist. Relation (5.1) below is a heuristic expression for $h_{*}$ as the depth halfway between HBL and the thermocline:

$$
h_{*}=\frac{1}{2}\left[\mathrm{HBL}+\max N^{2}(z)\right] .
$$

We constructed maps of $h_{*} / \mathrm{HBL}$ and $h_{*} / \mathrm{MLD}$, where MLD is computed using the potential density criterion $\Delta$ (potential density $)=0.03 \mathrm{~kg} \mathrm{~m}^{-3}$. We obtained the following results:

Maximum values: $h_{*} / \mathrm{HBL}=3.7, \quad h_{*} / \mathrm{MLD}=2.9$,

Mean values: $h_{*} / \mathrm{HBL}=1.5, \quad h_{*} / \mathrm{MLD}=1.2$,

which confirm the results of the numerical simulations of Mensa et al. (2013), Veneziani et al. (2014), Ramachandran et al. (2014), and Luneva et al. (2015) indicating that below the mixed layer the flow is still diabatic. Buckingham et al. (2017) also suggested use of the second term in (5.1), but for the first term, they used the MLD instead of the HBL.

\section{The OGCM}

We employed the 3D diffusivity tensor for an arbitrary tracer given in section 7 of $\mathrm{C} 18$, the mesoscale diffusivity (2.4), and the nonlocal version of the KPP vertical mixing scheme (Large et al. 1994) in the GISS ER model, which is the ocean component of the coupled NASA GISS model E (Russell et al. 1995, 2000; Liu et al. 2003). An early version of the revised E2-R
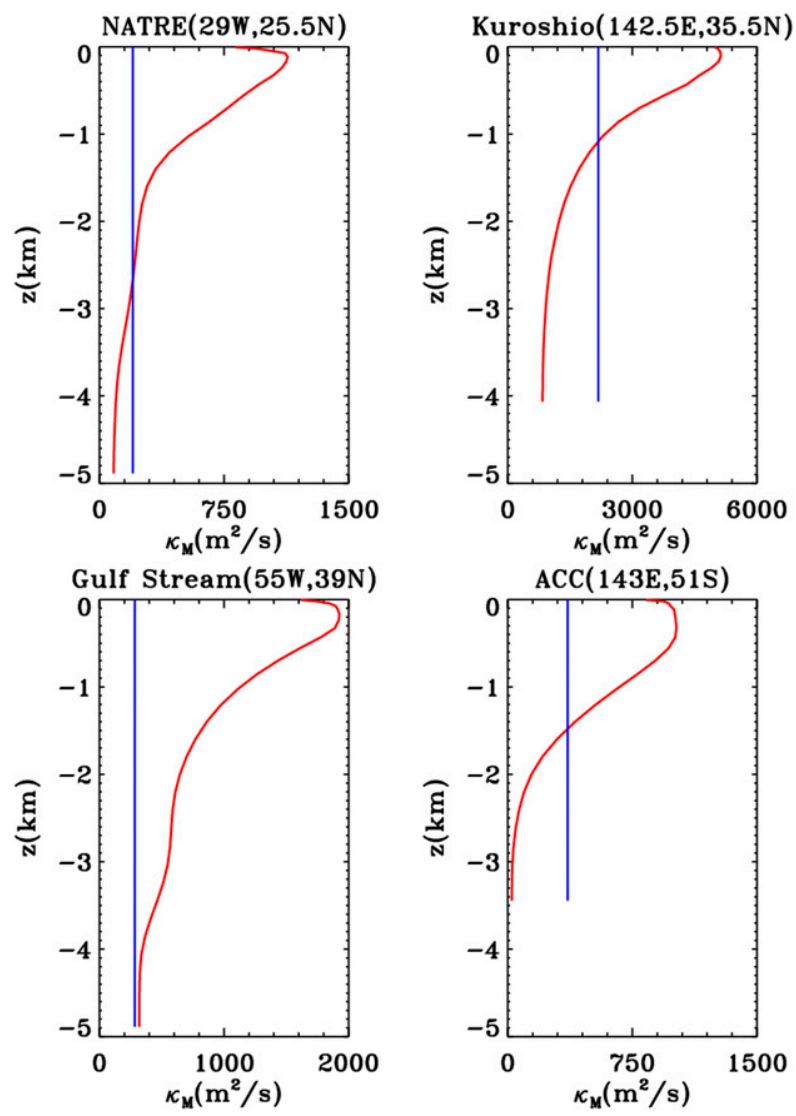

FIG. 6. Comparison of the 2D diffusivity $\kappa_{M}=0.13 \ell^{2}|f| \mathrm{Ri}^{-1 / 2}$ (Visbeck et al. 1997) (blue curves) with the present 3D model (2.5)-(2.6) (red curves).

code was run in a stand-alone mode (Danabasoglu et al. 2014). It employs a mass coordinate approximately proportional to pressure with 32 vertical layers with thickness from $\approx 12 \mathrm{~m}$ near the surface to $\approx 200 \mathrm{~m}$ at the bottom. The horizontal resolution is $1.25^{\circ}$ (longitude) by $1^{\circ}$ (latitude). It is a fully dynamic, non-Boussinesq, mass-conserving, free-surface ocean model using a quadratic upstream scheme for the horizontal advection of tracers and a centered difference scheme in the vertical. An 1800-s time step is used for tracer evolution. Sea ice dynamics, thermodynamics, and ocean-sea ice coupling are represented as in phase 5 of the Coupled Model Intercomparison Project (CMIP5) model-E configuration (Schmidt et al. 2014), save that here ice is on the ocean model grid. To force the model we used the Coordinated OceanIce Reference Experiment I (CORE-I) protocol (Griffies et al. 2009) with fluxes obtained from bulk formulas, the inputs to which are the ocean model surface state and atmospheric conditions derived from a synthesis of observations that repeat the seasonal cycle of a "normal year." The results we 

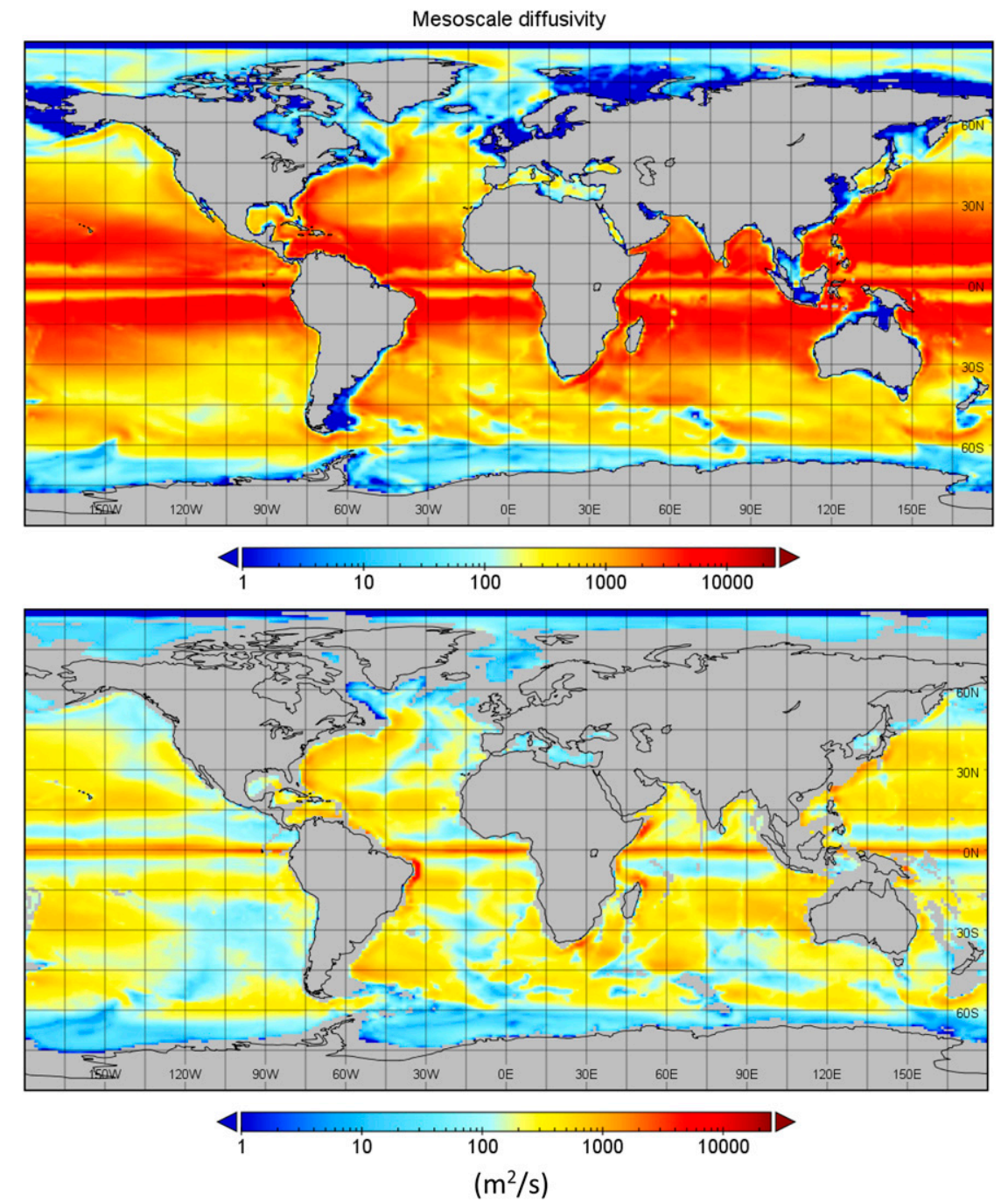

FIG. 7. Global maps of the diffusivity from (2.5)-(2.6) averaged over the 498th-500th years of the simulation: (top) 6-m depth and (bottom) 2003-m depth.

present in the next section correspond to the output of the final 3 years of a $300-y r$ run.

\section{Results}

Figures 2 and 3 exhibit surface values and the zonal average of the suppression factor $\varpi$ given by (2.6). Since no measurements or numerical simulations of this variable are available, the reason to exhibit its properties is to compare (2.6) with (2.7) of Bates et al. (2014). The key difference is that all the variables in (2.6) are predicted by the model while those in (2.7) are taken from observations with the results presented in their Figs. 5 and 8. In Fig. 2 near the equator, $\varpi$ shows the smallest values that become larger in the regions of the tropics of Cancer and Capricorn, followed by weaker minima in the temperate regions $40^{\circ}-50^{\circ}$ from the equator, before increasing again at high latitudes. The present model exhibits less small-scale variability than Fig. 5 of Bates et al. (2014), which may be due to the fact that in (2.7) the velocity $|\mathbf{c}|$ was determined in the ACC and then employed globally while in the present case the drift velocity from (A.2) depends on location. In Fig. 3 we show the latitude-depth plot of the zonally averaged $\varpi$ from (2.6). Suppression is strong at low latitudes whose limits progressively widen with depth, from about $10^{\circ}$ north and south of the equator at the surface to almost $30^{\circ}$ at the greatest depths. Compared with Fig. 8 of Bates et al. (2014), the present results are smoother, and the reason may be similar to the one suggested for Fig. 2. The present model does not show the sharp decrease of suppression 

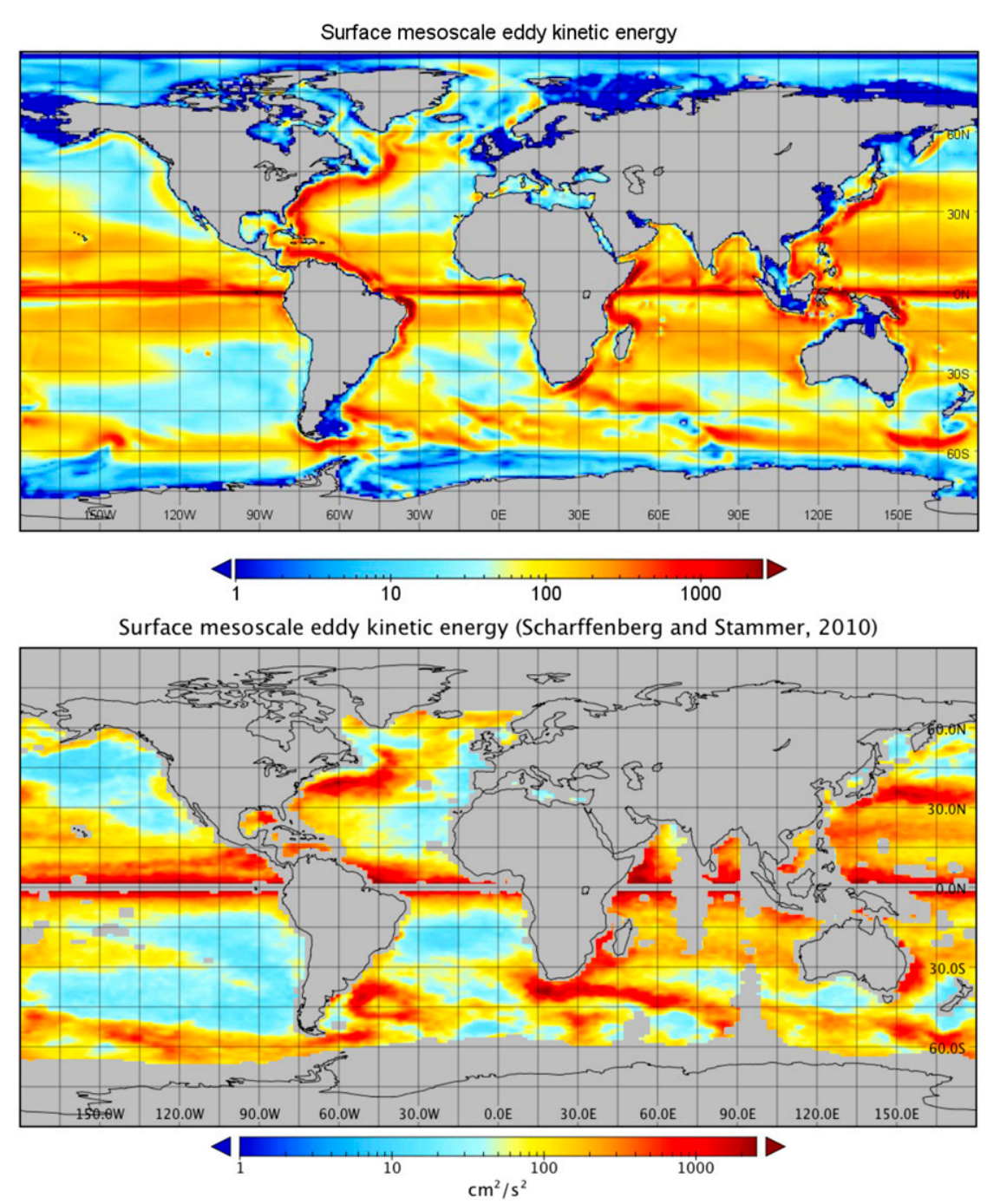

FIG. 8. (top) Map of the present model surface eddy kinetic energy $K_{s}[(4.7)]$. (bottom) Surface eddy kinetic energy from the T/P data (Scharffenberg and Stammer 2010).

near the equator that is prominent in Bates et al. (2014).

In Fig. 4 we show the depth profiles averaged between $61^{\circ}$ and $56^{\circ} \mathrm{S}$ and between $110^{\circ}$ and $80^{\circ} \mathrm{W}$ of the mean velocity (solid line), eddy drift velocity (dotted line), and square root of eddy kinetic energy (dashed line). If one compares these results with those in the lower panel of Fig. 10b of Tulloch et al. (2014), one notices several differences: the present eddy kinetic energy is steeper in the upper $200 \mathrm{~m}$, in Tulloch et al. (2014) the drift velocities $\mathbf{u}_{d}$ were computed using altimetry data while in the present work they are is given by (A.2), and the steering level in Tulloch et al. (2014) is at $1.6 \mathrm{~km}$ while in the present case it is at $2 \mathrm{~km}$. Finally, in Tulloch et al. (2014), $K$ was also computed using data while in the present case it is parameterized and later assessed as shown in Fig. 1 (as well as later in Fig. 8). One expected result in Fig. 4 is that the eddy kinetic energy is larger than that of the mean flow.

In Fig. 5 we show the vertical profile of the mesoscale diffusivity in (2.5)-(2.6) versus the NATRE data (Ferrari and Polzin 2005; Bates et al. 2014). The role of the suppression factor $\varpi<1$ is visible. The present model from (2.5) and (2.6) seems able to reproduce the data satisfactorily. The diffusivity model of Bates et al. (2014) is compared with the same data in their Fig. 10a.

In Fig. 6 we show the 2D diffusivity $\kappa_{M}=$ $0.13 \ell^{2}|f| \mathrm{Ri}^{-1 / 2}$ (blue lines; Visbeck et al. 1997) and the $3 \mathrm{D}$ form [(2.5) and (2.6)]: in all four locations, the present model yields significantly larger diffusivities in the upper $1 \mathrm{~km}$, and the $2 \mathrm{D}$ model has no vertical variation which may be relevant for the following reason. 


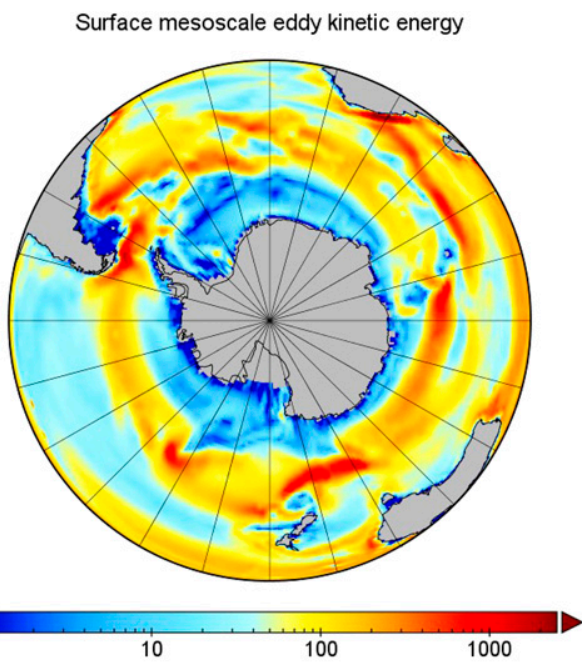

Surface mesoscale kinetic energy (Scharffenberg and Stammer, 2010)

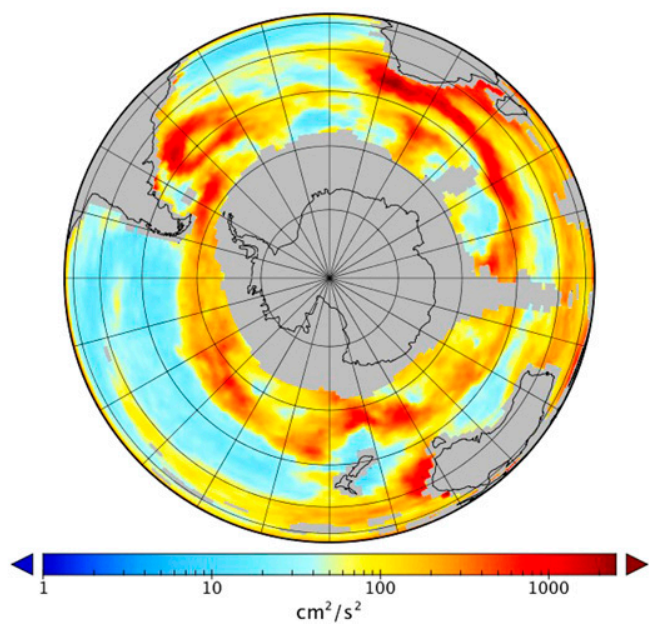

FIG. 9. (top) Map of the present model ACC surface eddy kinetic energy [(4.7)]. (bottom) The T/P data (Scharffenberg and Stammer 2010).

In a recent analysis, Kuhlbrodt and Gregory (2012, their Fig. 2a) showed that many of the 20 OGCMs exhibit weak stratification which they suggest may entail a large heat absorption in the deep ocean (see Cheng et al. 2017). A larger stratification would likely avoid this problem. The time evolution of $N^{2}$ is proportional ${ }^{2}$ to the negative second $z$ derivative of the vertical buoyancy flux which is proportional to the mesoscale diffusivity. Figure 6 shows that the 2D diffusivity used in many

\footnotetext{
${ }^{2}$ Consider the mean buoyancy equation, $D_{t} \bar{b}+\partial_{z} F_{v}(\bar{b})+$ $\nabla_{H} \cdot \mathbf{F}_{H}(\bar{b})=$ sources + sinks, where $F_{v}(\bar{b})$ and $\mathbf{F}_{H}(\bar{b})$ are the vertical and horizontal buoyancy fluxes, respectively. Taking the $z$ derivative of the equation and using $N^{2}=\partial_{z} \bar{b}$, one obtains the desired result.
}

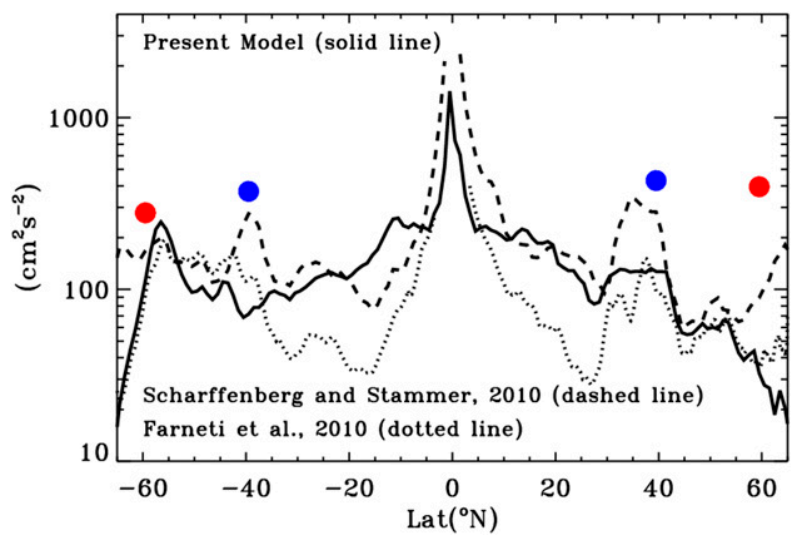

FIG. 10. Zonal average of the present model surface eddy kinetic energy [(4.7)] (solid line) vs T/P data (dashed line). The red and blue dots include the contributions of shear as described in (4.10). The dotted line represents the zonal average of the surface eddy kinetic energy from the numerical simulations of Farneti et al. (2010) using the CM2.4 version of the GFDL code.

OGCMs has no $z$ dependence and does not contribute to stratification. On the other hand, Fig. 9b of Luneva et al. (2015) shows that the present vertical buoyancy flux with 3D mesoscale diffusivity has a negative second $z$ derivative and enhances stratification, hopefully ameliorating the heat uptake problem discussed above.

Figure 7 shows global maps of the 3D mesoscale diffusivity from (2.5)-(2.6) averaged over the 298th-300th years of the simulation: 6-m depth (top panel) and 2-km

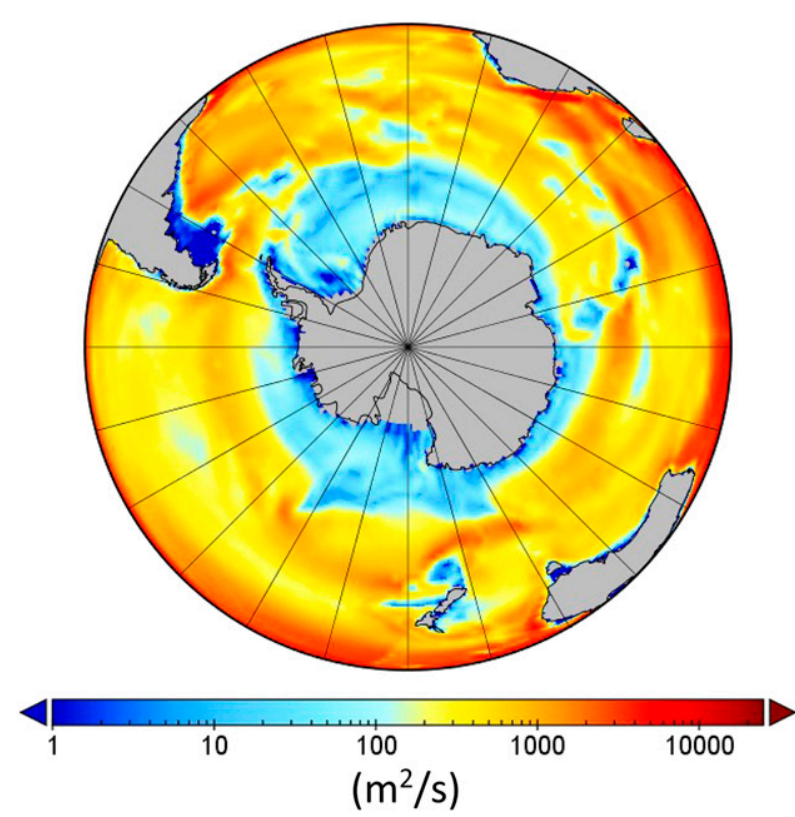

FIG. 11. Map of the ACC surface diffusivity from (2.5)-(2.6). The results compare well with Fig. 3f of Sallée et al. (2008) and Fig. 3 of Le Sommer et al. (2011). 

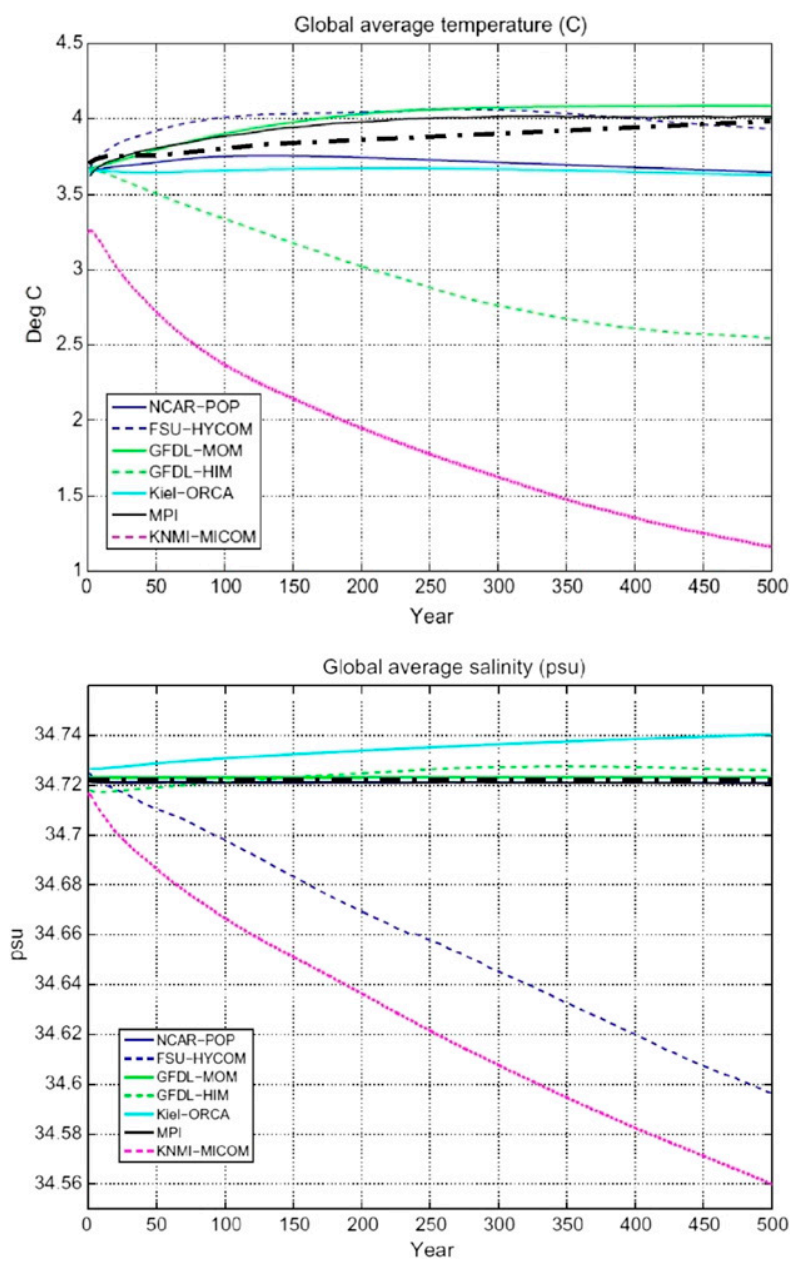

FIG. 12. Time series of globally and annually averaged ocean (top) potential temperature and (bottom) salinity with (2.5)-(2.6) (dash-dotted thick black curves) compared with the results of seven OGCMs shown in Figs. 3-4 of Griffies et al. (2009).

depth (bottom panel). The decrease with depth is an indication of the surface enhanced eddy kinetic energy shown in Fig. 1. The results can be compared with those of Fig. 8b of Klocker and Abernathey (2014).

The upper panel of Fig. 8 shows a map of $K_{s}(x, y)$ from (4.7) and the lower panel shows the T/P data (Scharffenberg and Stammer 2010). Considering that (4.7) is an analytic expression, the fact that it is capable of capturing the main features of $K_{s}(x, y)$ is a confirmation that baroclinic instabilities are a major contributor to the surface eddy kinetic energy.

The upper panel of Fig. 9 shows a map of the ACC surface eddy kinetic energy from (4.7), and the lower panel shows the corresponding $\mathrm{T} / \mathrm{P}$ data (Scharffenberg and Stammer 2010).

Figure 10 shows the zonal average of (4.7), the T/P data, and the results of a high-resolution numerical

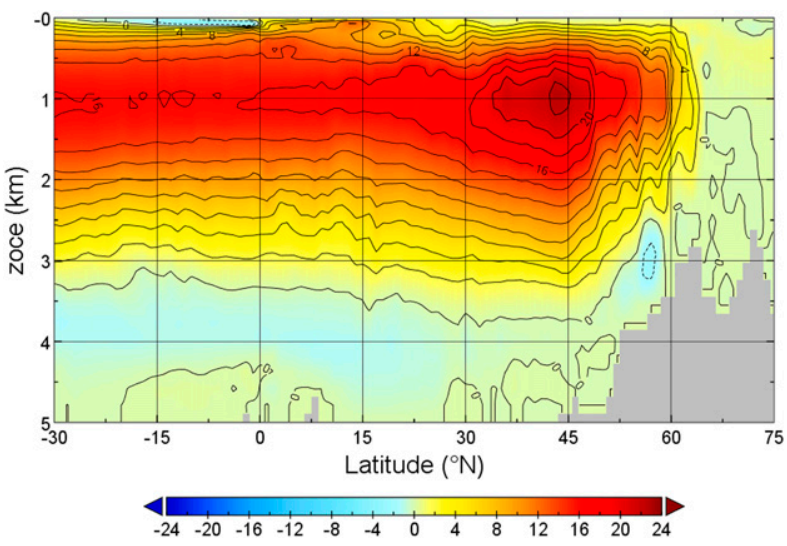

FIG. 13. Atlantic overturning circulation with (2.5)-(2.6). Results of seven OGCMs are shown in Fig. 23 of Griffies et al. (2009).

simulation by Farneti et al. (2010; CM2.4 version of the GFDL code, $1 / 4^{\circ}$ resolution, $27.75 \mathrm{~km}$ at the equator, $13.8 \mathrm{~km}$ at $60^{\circ} \mathrm{N}$, and $9 \mathrm{~km}$ at $70^{\circ} \mathrm{N} / \mathrm{S}$ ). These results are the only case in which we can compare model results with both T/P data and numerical simulations. On average, the simulation results are smaller than those of the present model that are close to the data. There is an instructive message: at $\pm 40^{\circ}$ and $\pm 60^{\circ}$ relation (4.7) underpredicts the surface eddy kinetic energy with respect to the T/P data. Though treated heuristically, the shear contribution in (4.10) represented by the blue and red dots brings the model results into better agreement with the T/P data.

Figure 11 shows the ACC surface diffusivity from (2.5)-(2.6), which compares well with the numerical simulations shown in Fig. 3f of Sallée et al. (2008) and in Fig. 3 of Le Sommer et al. (2011).

Figure 12 shows the time series of the globally and annually averaged temperature and salinity. Of the seven OGCMs results shown in Fig. 3 of Griffies et al. (2009), two exhibit a clear cooling tendency and one reaches stationarity only after 500 years while the other exhibits no tendency toward stationarity; the other five cases exhibit warming in time and reach stationarity after approximately 250 years. The result of the present model (black dash-dotted curve) exhibits a warming with a magnitude in the middle of the range of the other warming models. Of the seven OGCMs shown in Fig. 4 of Griffies et al. (2009), two have large fresh drifts, one has a moderate salty drift, and the rest have small drifts. The present model salinity drift is among the smallest.

In Fig. 13 we show the Atlantic meridional streamfunction $\left(1 \mathrm{~Sv}=10^{6} \mathrm{~m}^{3} \mathrm{~s}^{-1}\right)$ computed with the diffusivity from (2.5)-(2.6). The observational estimates are $16 \pm 2 \mathrm{~Sv}\left(48^{\circ} \mathrm{N}\right.$; Ganachaud 2003; Lumpkin et al. 2008), 

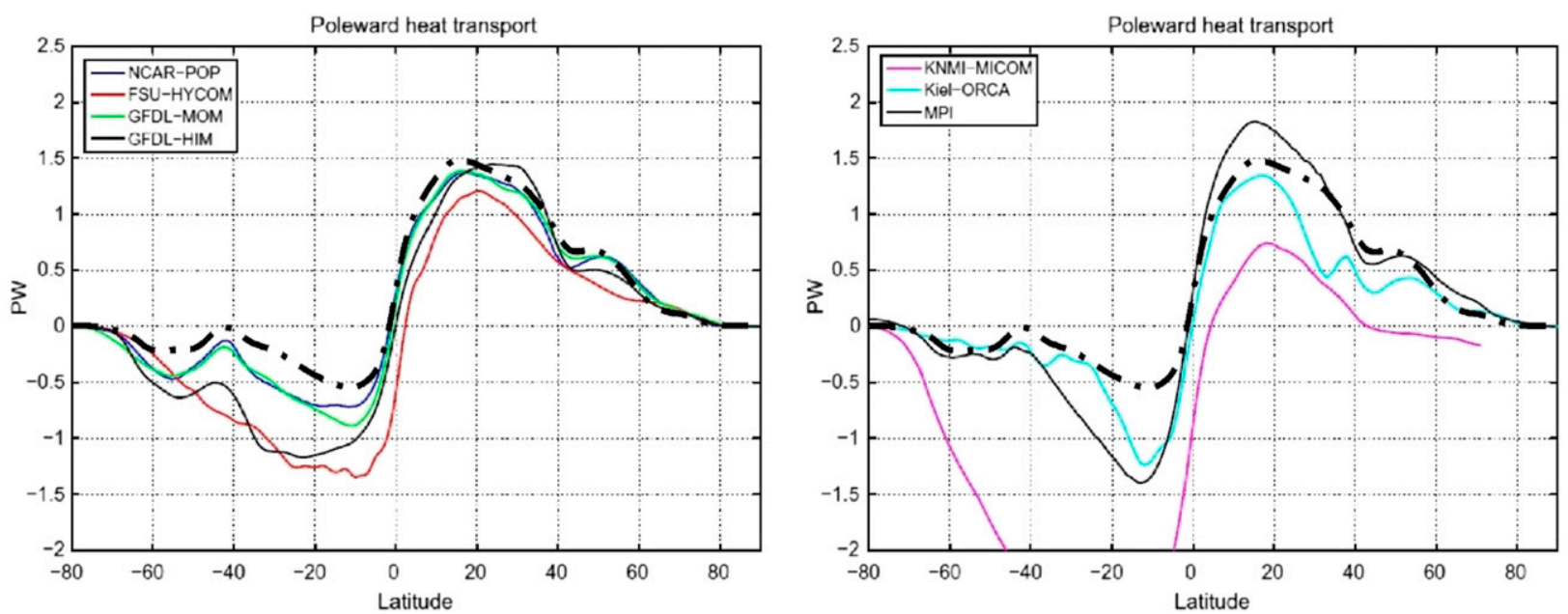

FIG. 14. Meridional heat transport in the global ocean averaged over the 491st-500th years of the simulation with (2.5)-(2.6) (dash-dotted thick black curve in each figure) compared with the results of seven OGCMs presented in Fig. 22 of Griffies et al. (2009).

$15 \pm 2 \mathrm{~Sv}\left(42^{\circ} \mathrm{N} ;\right.$ Ganachaud and Wunsch 2000$)$, and $13 \pm 2 \mathrm{~Sv}\left(42^{\circ} \mathrm{N}\right.$; Lumpkin and Speer 2003). Since this is a key oceanic feature, it is important to assess how well it is reproduced by different parameterizations. Of the seven OGCMs shown in Fig. 23 of Griffies et al. (2009), only three, the NCAR-POP, GFDL-MOM, and MPI OGCMs, yield results comparable to the data. The present model yields about $20 \mathrm{~Sv}$ within the observed values.

In Fig. 14 we show the meridional heat transport $\left(\mathrm{PW}=10^{15} \mathrm{~W}\right)$ in the global ocean averaged over the 298th-300th years of the simulation with (2.4) together with results of seven OGCMs from Fig. 22 of Griffies et al. (2009). Among the latter results there are discernable outliers while the present model result is well within the group of OGCMs that yield values of the order of $1 \mathrm{PW}$.

In Fig. 15 we present the vertically integrated mass transport through the Drake Passage with the diffusivity from (2.5)-(2.6) (dash-dotted thick black curve) compared with the results of seven OGCMs presented in Fig. 18 of Griffies et al. (2009) together with the observational data of $137 \pm 7.8 \mathrm{~Sv}$ (Cunningham et al. 2003). The spread of the results is rather large, and only two OGCMs seem capable of reproducing the observed data. The present model with the diffusivity from (2.5)-(2.6) reaches stationarity in less than 100 years.

Figure 16 (top panels) shows the 3-yr average mesoscale vertical buoyancy flux from C18 [their (2.3), (3.4a), and (3.6)] (blue lines), while the vertical buoyancy fluxes due to small-scale turbulence from the KPP scheme are in red. The positive mesoscale vertical buoyancy flux corresponds to upward heat transport, and the corresponding positive portion of the red curves is due to the nonlocality in the KPP buoyancy flux. The lower panels show the globally averaged temperature difference from the surface value, corresponding to the $3 \mathrm{D}$ versus the $2 \mathrm{D}$ mesoscale diffusivity $\kappa_{M}=0.13 \ell^{2}|f| \mathrm{Ri}^{-1 / 2}$ (Visbeck et al. 1997) that has no vertical structure. In the $3 \mathrm{D}$ case the results are close to the World Ocean Atlas 2005 data (Locarnini et al. 2006).

Figure 17 shows the temperature drift, a variable relevant to climate studies. Griffies et al. (2009) presented the temperature drifts corresponding to 14 OGCMs

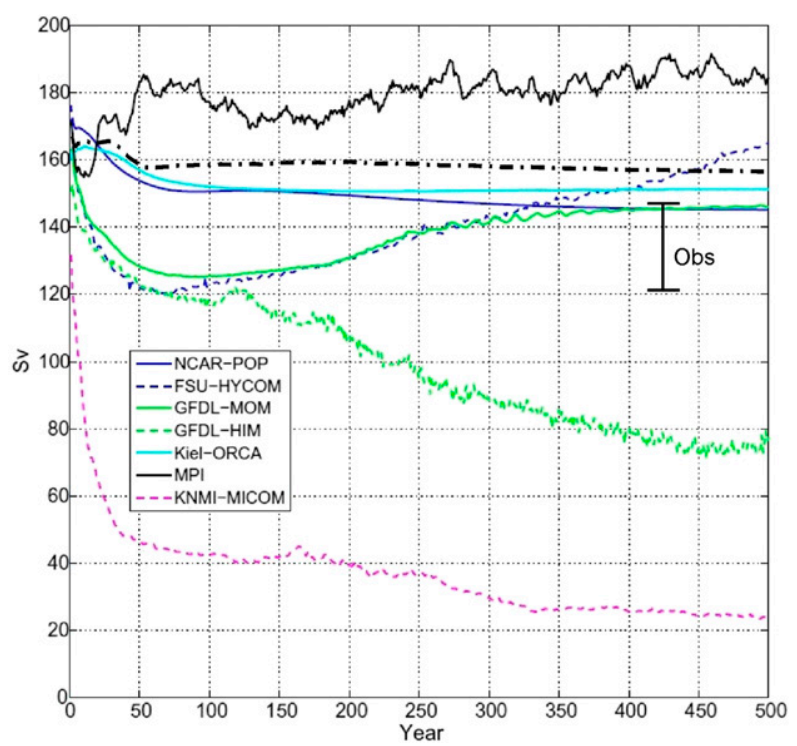

FIG. 15. Vertically integrated mass transport through the Drake Passage with (2.5)-(2.6) (dash-dotted thick black curve) compared with the results of seven OGCMs presented in Fig. 18 of Griffies et al. (2009) with the observational data of $137 \pm 7.8 \mathrm{~Sv}$ (Cunningham et al. 2003) 

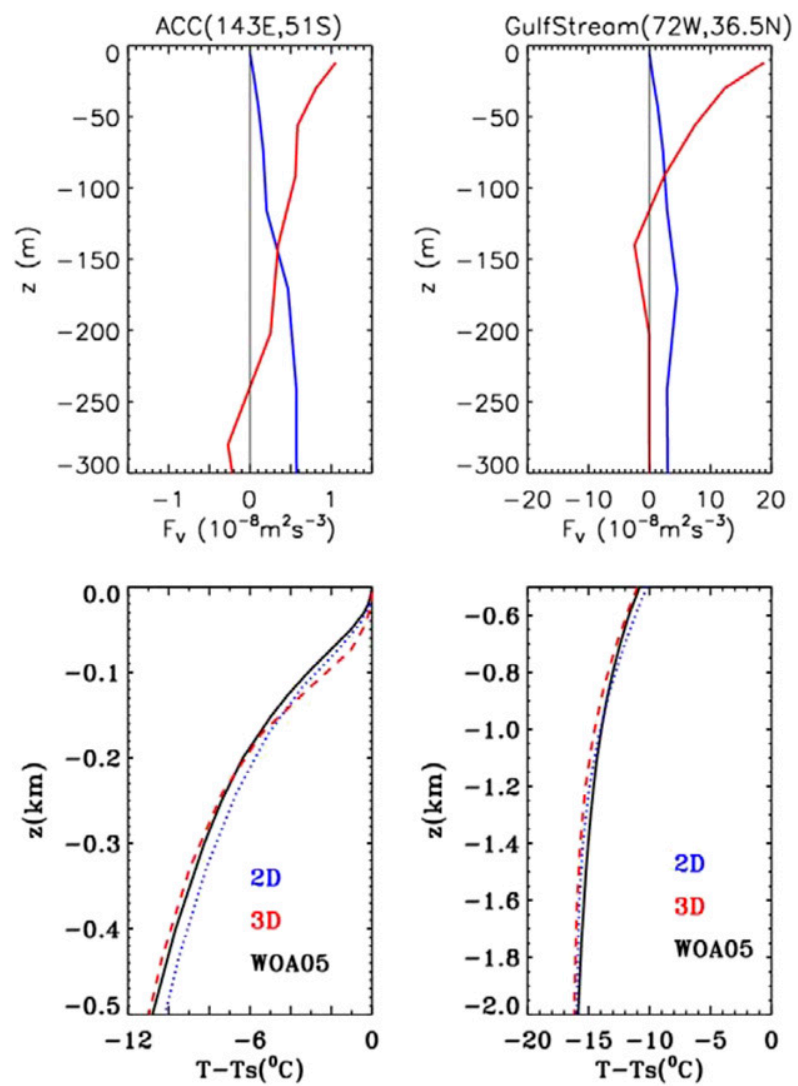

FIG. 16. (top) Annually averaged mesoscale vertical buoyancy flux from C18 [their (2.3), (3.4a), and (3.6)] (blue). The vertical buoyancy fluxes from small-scale turbulence from the KPP scheme are in red. The positive portion of the red curves is due to the presence of nonlocality in the buoyancy flux. (bottom) Globally averaged temperature difference from the surface value. The blue line corresponds to the $2 \mathrm{D}$ model results with the mesoscale diffusivity $\kappa_{M}=0.13 \ell^{2}|f| \mathrm{Ri}^{-1 / 2}$ (Visbeck et al. 1997). The red line represents the present $3 \mathrm{D}$ case, and the black line represents the WOA05 data (Locarnini et al. 2006).

(their Figs. 5 and 6) and concluded that "it is not trivial to uncover a mechanistic understanding of the drift patterns exhibited by the various models." In the present case, with the exception of the uppermost layers, in the bulk of the ocean the size of the drift is quite small, less than $1^{\circ} \mathrm{C}$.

Figure 18 shows that summer and winter ACC mixed layer depths MLD from the present model reproduce satisfactorily the data of Dong et al. (2008). It must be recalled that obtaining the correct MLD has not been easy, that is, of the seven OGCMs results in Fig. 15 of Griffies et al. (2009), only two reproduce the data. Boé et al. (2009) have emphasized the relation of MLD with the ocean heat uptake since models predicting deep mixed layers are transferring more of the radiative perturbation to the deep ocean, reducing surface

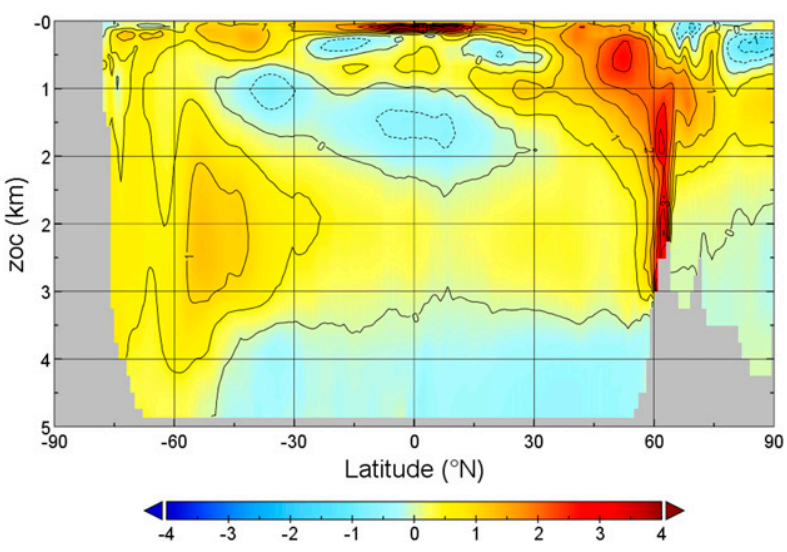

FIG. 17. Zonally averaged temperature minus observations $\left({ }^{\circ} \mathrm{C}\right)$ with (2.5)-(2.6).

warming. We recall that the MLD is the result of two competing processes, small scale turbulence that destratifies the flow yielding deep MLD and mesoscales that do the opposite, restratify the flow leading to a shallow MLD. We employed the KPP mixing scheme used by previous authors and changed the mesoscale model. Since the KKP mixing scheme contains the critical bulk Richardson number $\mathrm{Ri}_{b}(\mathrm{cr})$, which can vary between 0.3 and 1 , a mesoscale parameterization such as GM that induces lower stratification than the present model, may achieve satisfactory MLDs with a lower $\mathrm{Ri}_{b}(\mathrm{cr})$ while the present model that induces stronger stratification requires a larger $\mathrm{Ri}_{b}(\mathrm{cr})$. Specifically, with the GM model a value of $\mathrm{Ri}_{b}(\mathrm{cr})=0.3$ yields MLD similar to the ones we have obtained with $\mathrm{Ri}_{b}(\mathrm{cr})=1$.

\section{Conclusions and future work}

The primary goal of this work was to parameterize the 3D mesoscale diffusivity $\kappa_{M}$. While this was also the goal of Bates et al. (2014), Klocker and Abernathey (2014), and BA, there is an important difference with the present model. In those studies, the ingredients of $\kappa_{M}$, which are the mesoscale drift velocity and the kinetic energy, were not parameterized but determined using altimetry data, a procedure that deprives the models of the predictive power needed to study future climate unless one assumes that winds, stratification, etc., do not change in time, which is unlikely. In the present case, both ingredients are parameterized in terms of resolved variables and then assessed against available data, thus making the model predictive. The most difficult variable to parameterize was the surface $K_{s}(x, y)$, whose determination requires the vertical buoyancy flux that was presented in C18 and the Reynolds stresses for which we have 

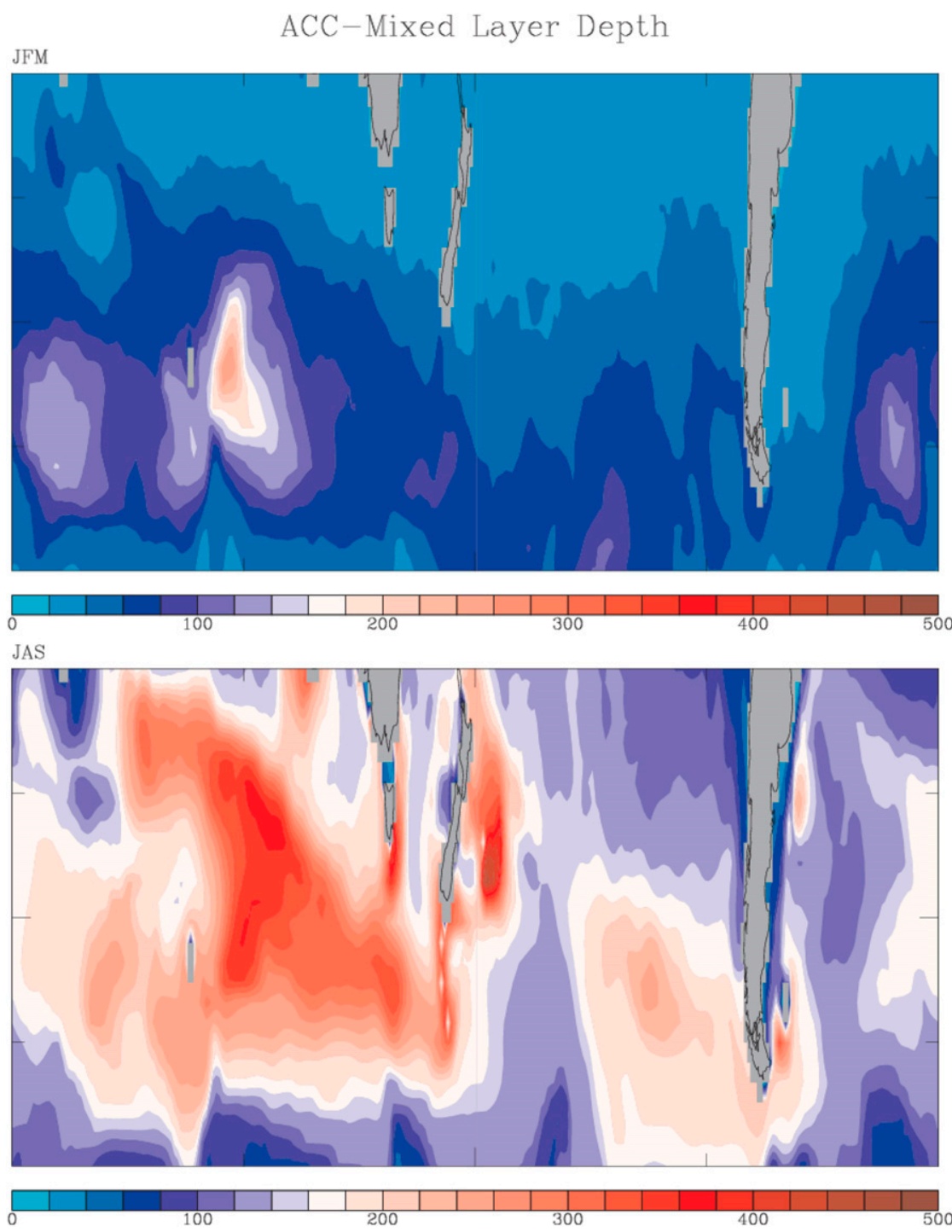

FIG. 18. ACC (top) summer and (bottom) winter mixed layer depths (m). The results reproduce satisfactorily the data by Dong et al. (2008).

no parameterization. In the future, we plan to address the following issues.

\section{a. Mesoscale Reynolds stresses}

The mesoscale Reynolds stresses enter the shear production term in (4.8) and represent the mesoscalemean flow interaction, which can be negative, representing eddies feeding the mean flow (e.g., in the Gulf Stream) and positive, representing the mean flow feeding eddies.

\section{b. Eddy compensation}

The eddy compensation process represents the response of the eddy field to an increase in the wind stress. It is relevant to climate studies (Bishop et al. 2016), since in its absence, deep ocean natural carbon can be brought to the surface hindering the absorption of atmospheric $\mathrm{CO}_{2}$. Eddy-resolving OGCMs have shown that mesoscales provide a partial compensating mechanism but coarse-resolution OGCMs have been less successful in reproducing it (Gent and Danabasoglu 2011; Farneti et al. 2015; Gent 2016; Poulsen et al. 2018). The hope is that the new 3D diffusivity will improve the skill of coarse-resolution OGCMs in reproducing the compensation process,

\section{c. Ocean heat uptake}

Gregory (2000) has pointed out that heat downward advection-upward diffusion, which is the reverse of a widely used model, may be a more appropriate model of 
oceanic heat transfer leading to the conclusion that the correct description of oceanic vertical heat transport processes is of "comparable importance" to the "climate sensitivity" in predicting climate change. Kuhlbrodt and Gregory (2012) showed that many ocean codes are too diffusive with a large capacity for downward heat transport [for a recent discussion of the 0-700-m and 700-2000-m heat content, see Cheng et al. (2017, e.g., their Fig. 6)]. The results shown in Fig. 16 indicate that a 3D mesoscale diffusivity entails a more stratified deep ocean thus hopefully avoiding excessive heat uptake. Prediction of the correct MLD is important to climate studies since models predicting deep mixed layers are transferring more of the radiative perturbation to the deep ocean, reducing surface warming (and vice versa).

Acknowledgments. The authors thank two anonymous referees whose valuable criticism helped us to achieve a more focused presentation. The authors also thank Dr. S. Dong for providing the Southern Ocean MLD data. Resources supporting this work were provided by the NASA High-End Computing (HEC) Program through the NASA Center for Climate Simulation (NCCS) at the Goddard Space Flight Center. VMC dedicates this work to Aura Sofia Canuto.

\section{APPENDIX A}

\section{Adiabatic-Diabatic Regimes}

\section{a. A regime}

In the A regime, water parcels move predominantly along surfaces of constant density (isopycnals) with negligible across-isopycnal excursions, that is, negligible diapycnal fluxes. The regime is commonly parameterized using the GM model (Gent and McWilliams 1990). The C18 parameterization accounts for features not present in the GM model that were highlighted by the mesoscale census of $\mathrm{C} 11$. These authors concluded that the westward propagating sea surface heights are dominated by "blobby structures" that are "coherent structures" that move with a translational or drift velocity $\mathbf{u}_{d}$ that does not coincide with the mean velocity $\overline{\mathbf{u}}$. C11 considered $\mathbf{u}_{d}$ the "most germane" of all the nonlinear metrics since "trapping of a fluid is the fundamental distinction between linear waves and non-linear eddies." By contrast, linear Rossby waves that initially are spatially compact lose their coherent structure because of dispersion, while mesoscale eddies conserve their identity for long distances and long periods of time making them relevant to climate. The form of $\mathbf{u}_{d}$ given in (A.2) below was compared with altimetry data in Fig. 1 of C18.
The presence of $\mathbf{u}_{d}$ alters the GM form of the eddyinduced velocity $\mathbf{u}^{+}$that we write in the form:

$$
\mathbf{u}^{+}=\mathbf{u}_{\mathrm{GM}}^{+}-\frac{\kappa_{M}}{f r_{d}^{2}} \mathbf{e}_{z} \times \mathbf{c}_{R}-\frac{\kappa_{M}}{\sigma f r_{d}^{2}} \mathbf{e}_{z} \times\left(\overline{\mathbf{u}}-\mathbf{u}_{d}\right)=-\frac{\partial \kappa_{M} \boldsymbol{\xi}}{\partial z} .
$$

Here, $\mathbf{u}_{\mathrm{GM}}^{+}=-\partial\left(\kappa_{M} \mathbf{s}\right) / \partial z, \mathbf{s}=-N^{-2} \nabla_{H} \bar{b}$ is the slope of the isopycnals, $b=-g \rho / \rho_{0}$ is the buoyancy, $\sigma \equiv$ $\sigma_{t}\left(1+\sigma_{t}\right)^{-1}, \sigma_{t}$ is the turbulent Prandtl number of $O(1)$, $\mathbf{e}_{z}=(0,0,1), \mathbf{c}_{R}=r_{d}^{2} \mathbf{e}_{z} \times \boldsymbol{\beta}$ is the Rossby phase velocity, $\boldsymbol{\beta}=\nabla f, f$ is the Coriolis parameter, and $r_{d}$ is the first Rossby deformation radius. The last form in (A.1) is a compact representation in which the vector $\boldsymbol{\xi}$ plays the role of a "dynamical slope" since it depends explicitly on the relative velocity $\overline{\mathbf{u}}-\mathbf{u}_{d}$, which requires a few comments. Killworth (1997) solved the linear mesoscale equations and obtained the first and second terms in (A.1); since he employed a linear model, the amplitude of the GM term could not be determined, only its structure. The novelty was the appearance of the second term representing the Rossby phase velocity $\mathbf{c}_{R}$ and playing the role of a drift velocity. However, since $\mathrm{C} 11$ showed that the drift velocity is the "most germane of all the non-linear metrics," it cannot be represented by the linear $\mathbf{c}_{R}$. Klocker and Marshall (2014) further showed that $\mathbf{c}_{R}$ does not reproduce the T/P data and suggested the addition of a vertically integrated $2 \mathrm{D}$ mean velocity $\overline{\mathbf{u}}$. The nonlinear model developed in CD5 yields (A.1) that includes $\mathbf{c}_{R}$, the mean velocity $\overline{\mathbf{u}}$, and the drift velocity $\mathbf{u}_{d}$. Because of Galilei translational invariance, $\overline{\mathbf{u}}$ and $\mathbf{u}_{d}$ appear as the relative velocity $\overline{\mathbf{u}}-\mathbf{u}_{d}$, which vanishes at the steering level where one recovers the GM-Killworth model. Since (A.2) shows that the first term in $\mathbf{u}_{d}$ is the Rossby phase velocity, in the final expression for $\mathbf{u}^{+}$, the linear term $\mathbf{c}_{R}$ cancels out and the main new term in (A.1) is $\overline{\mathbf{u}}-\langle\overline{\mathbf{u}}\rangle$, which reproduces the heuristic relation suggested by Klocker and Marshall (2014). The difference is that in the latter the average $\langle\ldots\rangle$ was a straight vertical average while in the nonlinear model, the average has a weight given by the mesoscale diffusivity [see (2.6) of C18]. As for the implications of this new term, in CD5 and in C18 (their sections $2 \mathrm{f}$ and $2 \mathrm{~g}$ ), it was shown that the first GM term in (A.1) yields a positive contribution to the energy transfer from large scales to mesoscales. Indeed, the GM term was suggested with the specific intent of representing a sink of mean potential energy. The $\overline{\mathbf{u}}-\mathbf{u}_{d}$ term yields instead a negative contribution to the energy exchange from large scales to mesoscales; this reduces the efficiency of the energy drawn by mesoscales, as shown in relation (2.15) of C18. This lower energy drawdown then means that the slope of the isopycnals is not as flat as in the GM case, as shown in Fig. 4a of C18. This feature 
may become relevant when studying the implications of the observed and predicted increase in the wind stress that tends to steepen the isopycnal slopes (Gent 2016).

\section{b. Drift velocity $\mathbf{u}_{d}$}

The drift velocity derived in $\mathrm{C} 18$ [their (2.5)] has the following form:

$\mathbf{u}_{d}(x, y)=\sigma \mathbf{c}_{R}+\langle\overline{\mathbf{u}}\rangle-\sigma f r_{d}^{2} \mathbf{e}_{z} \times\left(\left\langle\frac{\partial \mathbf{s}}{\partial z}\right\rangle-\frac{\mathbf{s}_{*}}{H_{*}}\right)$.

Here, the average $\langle\ldots\rangle$ defined in (2.6) of $\mathrm{C} 18$ is not a straight vertical average since it is weighted with $\kappa_{M}$, which is surface enhanced. The last term in (A.2) represents the contribution of the diabatic $\mathrm{D}$ regime, which was not accounted for in (5a) and (5b) of CD5 that was valid in an A regime only. The variable $\mathbf{s}_{*}$ represents the slope of the isopycnals at the A-D interface denoted by $h_{*}$ determined in section 5 ; the variable $H_{*}$ defined in (2.6) of $\mathrm{C} 18$ coincides with the ocean depth $H_{b}$ only in a linear model but not in a nonlinear case because of the presence of the mesoscale diffusivity in the vertical average. Using $r_{d}=10 \mathrm{~km},|\boldsymbol{\beta}|=10^{-11}\left(\mathrm{~m}^{-1} \mathrm{~s}^{-1}\right)$, the first (linear) term in (A.2) is on the order of $0.1 \mathrm{~cm} \mathrm{~s}^{-1}$; the second term is the largest of the three and $O(1-2) \mathrm{cm} \mathrm{s}^{-1}$, which is an order of magnitude larger than the first linear term. With $f=10^{-4} \mathrm{~s}^{-1}, r_{d}=10 \mathrm{~km}, s=10^{-3}$, $\partial_{z}=10^{-3} \mathrm{~m}^{-1}$, the third term is $O(1) \mathrm{cm} \mathrm{s}^{-1} \sigma$, which for $\sigma_{t}=0.72$ makes it somewhat smaller than the second term; the whole last term is negative and tends to lower the drift velocity. Figure 1 of $\mathrm{C} 18$ shows the comparison of (A.2) with $\mathrm{T} / \mathrm{P}$ altimetry data.

\section{c. D regime}

As discussed in $\mathrm{C} 18$, the parameterization of the $\mathrm{D}$ regime was not easy as attested by the several heuristic expressions that were proposed in the period 1999-2010. Since no model turned out to be superior to the others, many OGCMs adopted the simpler approach of prolonging the A-regime parameterization into the $\mathrm{D}$ regime using heuristic tapering functions. The latter is a numerical expedient rather than a physical model since in the D-regime water parcels no longer move along isopycnal surfaces as they do in the A regime and the diapycnal fluxes are large; furthermore, such an approach lacks predictive power and is therefore unsuitable to study future climate scenarios. In 2011, two of the authors (Canuto and Dubovikov 2011) used invariance principles and physical arguments to derive a D-regime parameterization that was assessed with a mesoscale resolving numerical simulation (Luneva et al. 2015). Two major projects, CMIP5 (Downes and Hogg 2013) and the CORE-I and -II simulations (Griffies et al. 2009;
Farneti et al. 2015), employed a variety of mesoscale parameterizations but none of them included the drift velocity $\mathbf{u}_{d}$.

\section{d. D-regime buoyancy flux}

For convenience of notation, we write the formula as follows:

$$
F_{v}(\bar{b})=-\hat{\mathbf{\kappa}} \cdot \nabla_{H} \bar{b}, \quad \hat{\mathbf{\kappa}}=\kappa_{M} \mathbf{\Omega},
$$

where the expression of $\boldsymbol{\Omega}(z)$ derived in $\mathrm{C} 18$ [their (3.2) and (3.4b)] has the form

$$
\boldsymbol{\Omega}(z)=\left[\boldsymbol{\omega}(z) \times \mathbf{e}_{z}-\Phi(z) \boldsymbol{\omega}_{*} \times \mathbf{e}_{z}\right]+\Phi(z) \frac{N(z)^{2}}{N_{*}^{2}} \mathbf{s}(z),
$$

where an asterisk means a variable at $z=-h_{*}$. Furthermore, the function $\Phi(z)$ must satisfy the boundary conditions $\Phi(0)=0, \Phi\left(-h_{*}\right)=1$ and has the form

$$
\Phi(\mathrm{z})=\frac{z^{2}}{h_{*}^{2}} \frac{N^{2}}{N_{*}^{2}}
$$

\section{APPENDIX B}

\section{Derivation of (2.6)}

We begin with the equation for the mesoscale buoyancy in $z$ coordinates (without source/sink):

$$
\partial_{t} b^{\prime}+\overline{\mathbf{U}} \cdot \nabla b^{\prime}+\mathbf{U}^{\prime} \cdot \nabla \bar{b}=Q, \quad Q=\overline{\mathbf{U}^{\prime} \cdot \nabla b^{\prime}}-\mathbf{U}^{\prime} \cdot \nabla b^{\prime},
$$

where $\mathbf{U}(\overline{\mathbf{u}}, w)$ is the $3 \mathrm{D}$ mean velocity field, $\nabla$ is the $3 \mathrm{D}$ gradient operator, and $Q$ is the nonlinear term. In the vicinity of the surface we can neglect the vertical velocity component. Under this condition, we Fourier transform (B.1), which implies that the length and time scales of mean fields are much larger than of mesoscale eddies. In other words, in a Fourier transform, the flow is considered quasi stationary and quasi homogeneous, that is, the mean fields are assumed to be constant. Then, in Fourier space, we have [CD5, their (15a)]

$$
\nabla_{H} b^{\prime}=i \mathbf{k} b^{\prime}, \quad \partial_{\mathrm{t}} b^{\prime}=-i \omega b^{\prime}, \quad \omega=\mathbf{k} \cdot \mathbf{u}_{d},
$$

where $\nabla_{H}$ is the horizontal component of $\nabla$. As for the nonlinear term $Q$, the model presented in CD5 yields the following result in the vicinity of the maximum of the energy spectrum $|\mathbf{k}|=r_{d}^{-1}$ :

$$
Q=-\chi b^{\prime}, \quad \chi=r_{d}^{-1} K^{1 / 2} .
$$


Substituting (B.2) and (B.3) into (B.1), one obtains

$$
b^{\prime}(\mathbf{k})=-\left(i \mathbf{k} \cdot \mathbf{u}_{D}+\chi\right)^{-1} \mathbf{u}^{\prime}(\mathbf{k}) \cdot \nabla_{H} \bar{b}, \quad \mathbf{u}_{D}=\overline{\mathbf{u}}-\mathbf{u}_{d} .
$$

To find the horizontal buoyancy flux, we multiply (B.4) by $\mathbf{u}^{\prime *}\left(\mathbf{k}^{\prime}\right)$ and average the result over the ensemble of mesoscale fields realizations. From the homogeneous nature of mesoscale turbulence, it follows the definition for the horizontal buoyancy flux in wave-vector space $\mathbf{F}_{H}(\mathbf{k})=\overline{\mathbf{u}^{\prime} b^{\prime}}(\mathbf{k})$ :

$$
\begin{aligned}
\mathbf{F}_{H} & \equiv \overline{\mathbf{u}^{\prime} b^{\prime}}=\int d^{2} \overline{\mathbf{k}} \overline{\mathbf{u}^{\prime} b^{\prime}}(\mathbf{k}), \quad \overline{\bar{u}^{\prime} b^{\prime}}(\mathbf{k}) \delta\left(\mathbf{k}-\mathbf{k}^{\prime}\right) \\
& =\operatorname{Re} \overline{\mathbf{u}^{\prime *}(\mathbf{k}) b^{\prime}\left(\mathbf{k}^{\prime}\right)} .
\end{aligned}
$$

Assuming that the mesoscale energy spectrum is concentrated in the vicinity of its maximum at $|\mathbf{k}|=r_{d}^{-1}$ and that the mesoscale velocity field is isotropic, that is, $\overline{u_{\alpha}^{\prime} u_{\beta}^{\prime}}=K \delta_{\alpha \beta}$, after ensemble averaging using the second relation in (B.3), one obtains

$$
\begin{gathered}
\mathbf{F}_{H}=-\kappa_{M} \nabla_{H} \bar{b}, \quad \kappa_{M}=r_{d} K^{1 / 2} \varpi \\
\varpi=\int d \mathbf{n}\left[1+K^{-1}\left(\mathbf{n} \cdot \mathbf{u}_{D}\right)^{2}\right]^{-1}
\end{gathered}
$$

After integration in (B.6), one obtains

$$
\varpi=\left[1+\frac{1}{K}\left|\mathbf{u}_{D}\right|^{2}\right]^{-1 / 2},
$$

which, to the main order in $K^{-1}\left|\mathbf{u}_{D}\right|^{2}$, becomes relation (2.6):

$$
\varpi=\left(1+\frac{1}{2 K}\left|\mathbf{u}_{D}\right|^{2}\right)^{-1}
$$

\section{REFERENCES}

Bates, M., R. Tulloch, J. Marshall, and R. Ferrari, 2014: Rationalizing the spatial distribution of mesoscale eddy diffusivity in terms of mixing length theory. J. Phys. Oceanogr., 44, 15231540, https://doi.org/10.1175/JPO-D-13-0130.1.

Bi, D., W.F. Budd, A.C. Hirst and X. Wu, 2002: Response of the Antarctic Circumpolar Current transport to global warming in a coupled model. Geophys. Res. Lett., 29, 2173, https:// doi.org/10.1029/2002GL015919.

Bishop, S., P. R. Gent, F. O. Bryan, A. F. Thompson, M. C. Long, and R. Abernathey, 2016: Southern Ocean overturning compensation in an eddy-resolving climate simulation. J. Phys. Oceanogr., 46, 1575-1592, https://doi.org/10.1175/ JPO-D-15-0177.1.

Boé, J., A. Hall and X. Qu, 2009: Deep ocean heat uptake as a major source of spread in transient climate change simulations, Geophys. Res. Lett., 36, L22701, https://doi.org/10.1029/ 2009GL040845.
Boning, C. W., A. Dispert, M. Visbeck, S. R. Rintoul, and F. U. Schwarzkopf, 2008: The response of the Antarctic Circumpolar Current to recent climate change. Nat. Geosci., 1, 864-869, https://doi.org/10.1038/ngeo362.

Bryan, F., P. R. Gent, and R. Tomas, 2014: Can Southern Ocean eddy effects be parameterized in climate models? J. Climate, 27, 411-425, https://doi.org/10.1175/JCLI-D-12-00759.1.

Bryden, H. L., and E. C. Brady, 1989: Eddy momentum and heat fluxes and their effect on the circulation of the equatorial Pacific Ocean. J. Mar. Res., 47, 55-79, https://doi.org/10.1357/ 002224089785076389.

Buckingham, C. E., and Coauthors, 2017: Testing Munk's hypothesis for sub-mesoscale eddy generation using observations in the North Atlantic. J. Geophys. Res. Oceans, 122, 6725-6745, https://doi.org/10.1002/2017JC012910.

Busecke, J. J. M., and R. P. Abernathey, 2019: Ocean mesoscale mixing linked to climate variability. Sci. $A d v$., 5, eaav5014, https://doi.org/10.1126/sciadv.aav5014.

Canuto, V. M., and I. Mazzitelli, 1991: Stellar turbulent convection: New model and applications. Astrophys. J., 370, 295-311.

, and M. S. Dubovikov, 1996: A dynamical model for turbulence. I. General formalism. Phys. Fluids, 8, 571-586, https:// doi.org/10.1063/1.868842.

- and - 2005: Modeling mesoscale eddies. Ocean Modell., 8, 1-30, https://doi.org/10.1016/j.ocemod.2003.11.003.

—_, and —-, 2006: Dynamical model of mesoscales in z-coordinates. Ocean Modell., 11, 123-166, https://doi.org/ 10.1016/j.ocemod.2004.12.002.

— scale parameterizations and the equation for an arbitrary tracer. Ocean Modell., 39, 200-207, https://doi.org/10.1016/ j.ocemod.2011.04.008.

— G. J. Hartke, A. Battaglia, and J. Chasnov, 1990: Theoretical study of turbulent channel flow: Bulk properties, pressure fluctuations, and propagation of electromagnetic waves. J. Fluid Mech., 211, 1-17, https://doi.org/10.1017/S0022112090001471.

—, Y. Cheng, M. S. Dubovikov, A. M. Howard, and A. Leboissetier, 2018: Parameterization of mixed layer and deep-ocean mesoscales including nonlinearity. J. Phys. Oceanogr., 48, 555-572, https://doi.org/10.1175/JPO-D-16-0255.1.

Cessi, P., 2008: An energy-constrained parameterization of eddy buoyancy flux. J. Phys. Oceanogr., 38, 1807-1819, https:// doi.org/10.1175/2007JPO3812.1.

Charney, J. G., 1971: Geostrophic turbulence. J. Atmos. Sci., 28, 1087-1095, https://doi.org/10.1175/1520-0469(1971)028<1087: $\mathrm{GT}>2.0 . \mathrm{CO} ; 2$.

Chelton, D. B., M. G. Schlax, and R. M. Samelson, 2011: Global observations of non-linear mesoscale eddies. Prog. Oceanogr., 91, 167-216, https://doi.org/10.1016/j.pocean.2011.01.002.

Cheng, L., K. E. Trenberth, J. Fasullo, T. Boyer, J. Abraham, and J. Zhu, 2017: Improved estimates of ocean content from 1960 to 2015. Sci. Adv., 3, e1601545, https://doi.org/10.1126/sciadv.1601545.

Cunningham, S. A., S. G. Alderson, B. A. King, and M. A. Brandon, 2003: Transport and variability of the Antarctic Circumpolar Current in the Drake Passage. J. Geophys. Res., 108, 8084, https://doi.org/10.1029/2001JC001147.

Danabasoglu, G., and J. Marshall, 2007: Effects of vertical variations of thickness diffusivity in an ocean general circulation model. Ocean Modell., 18, 122-141, https://doi.org/10.1016/j.ocemod.2007.03.006. , and Coauthors, 2014: North Atlantic simulations in Coordinated Ocean-ice reference experiment phase II (COREII). Part I: Mean states. Ocean Modell., 73, 76-107, https:// doi.org/10.1016/j.ocemod.2013.10.005. 
Danilov, S. D., and D. Gurarie, 2000: Quasi-2D turbulence. Phys. Usp., 43, 863-900, https://doi.org/10.1070/PU2000v043n09ABEH000782.

Dong, S., J. Sprintall, S. T. Gille, and L. Talley, 2008: Southern Ocean mixed-layer depth from Argo float profiles. J. Geophys. Res., 113, C06013, https://doi.org/10.1029/2006JC004051.

Downes, S. M., and A. McC. Hogg, 2013: Southern Ocean circulation and eddy compensation in CMIP5 models. J. Climate, 26, 7198-7220, https://doi.org/10.1175/JCLI-D12-00504.1.

Eden, C., and R. J. Greatbatch, 2008: Towards a mesoscale eddy closure. Ocean Modell., 20, 223-239, https://doi.org/10.1016/ j.ocemod.2007.09.002.

Farneti, R., and P. R. Gent, 2011: The effect of the eddy-induced advection coefficient in a coarse resolution coupled climate model. Ocean Modell., 39, 135-145, https://doi.org/10.1016/ j.ocemod.2011.02.005.

_ T. L Delworth, A. J. Rosati, S. M. Griifies, and F. Zeng, 2010: The role of mesoscale eddies in the re-stratification of the Souter Ocean response to climate change. J. Phys. Oceanogr., 40, 1539-1557, https://doi.org/10.1175/2010JPO4353.1.

_ - and Coauthors, 2015: An assessment of Antarctic Circumpolar Current and Southern Ocean meridional overturning circulation during 1958-2007 in a suite of interannual COREII simulations. Ocean Modell., 93, 84-120, https://doi.org/ 10.1016/j.ocemod.2015.07.009.

Ferrari, R., and K. I. Polzin, 2005: Finescale structure of the $T-S$ relation in the eastern North Atlantic. J. Phys. Oceanogr., 35 1437-1454, https://doi.org/10.1175/JPO2763.1.

_ , and M. Nikurashin, 2010: Suppression of eddy diffusivity across jets in the Southern Ocean. J. Phys. Oceanogr., 40, 1501-1519, https://doi.org/10.1175/2010JPO4278.1.

Ferreira, D., J. Marshall, and P. Heimbach, 2005: Estimating eddy stresses by fitting dynamics to observations using a residual-mean ocean circulation model and its adjoint. J. Phys. Oceanogr., 35, 1891-1910, https://doi.org/10.1175/ JPO2785.1.

Frenger, I., M. Munnich, N. Gruber, and R. Knutti, 2015: Southern Ocean eddy phenomenology. J. Geophys. Res., 120, 74137449, https://doi.org/10.1002/2015JC011047.

Fu, L.-L., 2009: Pattern and velocity of propagation of the global ocean eddy variability. J. Geophys. Res., 114, C11017, https:// doi.org/10.1029/2009JC005349.

Ganachaud, A., 2003: Large-scale mass transport, water mass formation and diffusivities estimated from World Ocean Circulation Experiment (WOCE) hydrographic data. J. Geophys. Res., 108, 3213, https://doi.org/10.1029/ 2002JC001565.

— , and C. Wunsch, 2000: Improved estimates of global circulation, heat transport and mixing from hydrographic data. Nature, 408, 453-456, https://doi.org/10.1038/35044048.

Gent, P. R., 2011: The Gent-McWilliams parameterization: 20/20 hindsight. Ocean Modell., 39, 2-9, https://doi.org/10.1016/ j.ocemod.2010.08.002.

_ - 2016: Effects of Southern Hemisphere wind changes on the meridional overturning circulation in ocean models. Annu Rev. Mar. Sci., 8, 79-94, https://doi.org/10.1146/annurev-marine122414-033929.

- and J. C. McWilliams, 1990: Isopycnal mixing in ocean circulation models. J. Phys. Oceanogr., 20, 150-155, https:// doi.org/10.1175/1520-0485(1990)020<0150:IMIOCM>2.0.CO;2.

_ _ and G. Danabasoglu, 2011: Response to increasing Southern Hemisphere winds in CCSM4. J. Climate, 24, 4992-4998, https://doi.org/10.1175/JCLI-D-10-05011.1.
Gill, A. E., J. S. A. Green, and A. J. Simmons, 1974: Energy partition in the large-scale circulation and the production of midocean eddies. Deep-Sea Res. Oceanogr. Abstr., 21, 499-528, https://doi.org/10.1016/0011-7471(74)90010-2.

Gregory, J. M., 2000: Vertical heat transports in the ocean and their effect on time dependent climate change. Climate Dyn., 16, 501-515, https://doi.org/10.1007/s003820000059.

Griffies, S. M., and Coauthors, 2009: Coordinated Ocean-ice Reference Experiments (COREs). Ocean Modell., 26, 1-46, https://doi.org/10.1016/j.ocemod.2008.08.007.

Hofman, M., and M. A. Morales Maqueda, 2011: The response of Southern Ocean eddies to increased mid-latitude westerlies: A non-eddy resolving study. Geophys. Res. Lett., 38, L03605, https://doi.org/10.1029/2010GL045972.

Killworth, P. D., 1997: On parameterization of eddy transport. J. Mar. Res., 55, 1171-1197, https://doi.org/10.1357/0022240973224102.

Klocker, A., and R. Abernathey, 2014: Global patterns of mesoscale eddy properties and diffusivities. J. Phys. Oceanogr., 44, 1030-1046, https://doi.org/10.1175/JPO-D13-0159.1.

— , and D. P. Marshall, 2014: Advection of baroclinic eddies by depth mean flow. Geophys. Res. Lett., 41, 3517-3521, https:// doi.org/10.1002/2014GL060001.

Kuhlbrodt, T., and J. M. Gregory, 2012: Ocean heat uptake and its consequences for the magnitude of sea level rise and climate change. Geophys. Res. Lett., 39, L18608, https://doi.org/ 10.1029/2012GL052952.

, R. S. Smith, Z. Wang, and J. M. Gregory, 2012: The influence of eddy parameterizations on the transport of the Antarctic Circumpolar Current in coupled climate models. Ocean Modell., 52-53, 1-8, https://doi.org/10.1016/j.ocemod.2012.04.006.

Large, W. G., J. C. McWilliams, and S. C. Doney, 1994: Oceanic vertical mixing: A review and a model with a nonlocal boundary layer parameterization. Rev. Geophys., 32, 363-403, https://doi.org/10.1029/94RG01872.

—, G. Danabasoglu, and S. C. Doney, 1997: Sensitivity to surface forcing and boundary layer mixing in a global ocean model: Annual-mean climatology. J. Phys. Oceanogr., 27, 2418-2447, https://doi.org/10.1175/1520-0485(1997)027<2418:STSFAB > 2.0.CO;2.

Lebedev, K. V., H. Yoshinari, N. A. Maximenko, and P. W. Hacker, 2007: YoMaHa'07: Velocity data assessed from trajectories of Argo floats at parking level and at the sea surface. IPRC Tech. Note 4(2), 20 pp., http://apdrc.soest.hawaii.edu/ projects/yomaha/yomaha07/YoMaHa070612.pdf.

Le Sommer, J., F. d'Ovidio, and G. Madec, 2011: Parameterization of subgrid stirring in eddy resolving ocean models. Part I: Theory and diagnostics. Ocean Modell., 39, 154-169, https:// doi.org/10.1016/j.ocemod.2011.03.007.

Liu, J., G. A. Schmidt, D. G. Martinson, D. Rind, G. L. Russell, and X. Yuan, 2003: Sensitivity of sea ice to physical parameterizations in the GISS global climate model. J. Geophys. Res., 108, 3053, https://doi.org/10.1029/2001JC001167.

Locarnini, R. A., A. V. Mishonov, J. I. Antonov, P. T. Boyer, and H. E. Garcia, 2006, Temperature. Vol. 1, World Ocean Atlas 2005, NOAA Atlas NESDIS 61, 182 pp.

Lumley, J. L., and A. Yaglom, 2001: A century of turbulence. Flow Turbul. Combust., 66, 241-286, https://doi.org/10.1023/ A:1012437421667.

Lumpkin, R., and K. Speer, 2003: Large scale vertical and horizontal circulation in the North Atlantic Ocean. J. Phys. Oceanogr., 33, 1902-1920, https://doi.org/10.1175/1520-0485(2003) $033<1902:$ LVAHCI $>2.0$. CO;2. 
- - - - and K. Kolterman, 2008: Transport across $28^{\circ} \mathrm{N}$ in the Atlantic Ocean. J. Phys. Oceanogr., 38, 733-752, https:// doi.org/10.1175/2007JPO3636.1.

Luneva, M. V., C. A. Clayson, and M. S. Dubovikov, 2015: Effects of mesoscale eddies in the active mixed layer: Test of the parameterization in eddy resolving simulations. Geophys. Astrophys. Fluid Dyn., 109, 281-310, https://doi.org/10.1080/ 03091929.2015.1041023.

McComb, W. D., 1992: The Physics of Fluid Turbulence. Oxford Science Publications, 572 pp.

Mensa, J. A., Z. Garraffo, A. Griffa, T. M. Ozgokmen, A. Haza, and M. Veneziani, 2013: Seasonality of sub-mesoscale dynamics in the Gulf Stream region. Ocean Dyn., 63, 923-941, https:// doi.org/10.1007/s10236-013-0633-1.

Meredith, M. P., A. C. Naveira Garabato, A. Mc.Hogg, and R. Farneti, 2012: Sensitivity of the overturning circulation in the southern ocean to decadal changes in wind forcing. J. Climate, 25, 99-110, https://doi.org/10.1175/2011JCLI4204.1.

Poulsen, M. B., M. Jochum, and R. Nuterman, 2018: Parameterized and resolved Southern Ocean eddy compensation. Ocean Modell., 124, 1-15, https://doi.org/10.1016/j.ocemod.2018.01.008.

Prandtl, L., 1925: Bericht uber Untersuchungen zur ausgebildeten Turbulenz. Z. Angew. Math. Mech., 5, 136-139.

Ramachandran, S., A. Tandon, and A. Mahadevan, 2014: Enhancement in vertical fluxes at a front by mesoscalesubmesoscale coupling. J. Geophys. Res. Oceans, 119, 84958511, https://doi.org/10.1002/2014JC010211.

Roach, C. J., D. Balwada, and K. Speer, 2018: Global observations of horizontal mixing from Argo floats and surface drifter trajectories. J. Geophys. Res. Oceans, 123, 4560-4575, https:// doi.org/10.1029/2018JC013750.

Robinson, A. R., and J. C. McWilliams, 1974: The baroclinic instability of the open ocean. J. Phys. Oceanogr., 4, 281-294, https://doi.org/ 10.1175/1520-0485(1974)004<0281:TBIOTO > 2.0.CO;2.

Russell, G. L., J. R. Miller, and D. H. Rind, 1995: A coupled atmosphere-ocean model for transient climate change. Atmos.Ocean, 33, 683-730, https://doi.org/10.1080/07055900.1995.9649550.

,,,--- R. A. Ruedy, G. A. Schmidt, and S. Sheth, 2000: Comparison of model and observed regional temperature changes during the past 40 +years. J. Geophys. Res., 105, 14 891-14 898, https://doi.org/10.1029/2000JD900156.

Saenko, O. A., J. C. Fyfe, and M. H. England, 2005: On the response of the ocean wind-driven circulation to atmospheric $\mathrm{CO}_{2}$ increase. Climate Dyn., 25, 415-426, https://doi.org/ 10.1007/s00382-005-0032-5.

Sallée, J. B., K. Speer, R. Morrow, and R. Lumpkin, 2008: An estimate of Lagrangian eddy statistics and diffusion in the mixed layer of the Southern Ocean. J. Mar. Res., 66, 441-463., https:// doi.org/10.1357/002224008787157458.

Scharffenberg, M. G., and D. Stammer, 2010: Seasonal variations of the geostrophic flow field and of eddy kinetic energy inferred from TOPEX/Poseidon and Jason-1 Tandem Mission Data. $J$. Geophys. Res., 115, C02008, https://doi.org/10.1029/2008JC005242.

Schmidt, G. A., and Coauthors, 2014: Configuration and assessment of the GISS ModelE2 contributions to the CMIP5 archive. J. Adv. Model. Earth Syst., 6, 141-184, https://doi.org/ 10.1002/2013MS000265.

Scott, R. B., and F. Wang, 2005: Direct evidence of an oceanic inverse kinetic energy cascade from satellite altimetry. J. Phys. Oceanogr., 35, 1650-1666, https://doi.org/10.1175/JPO2771.1.

Smith, K. S., 2007: The geography of linear baroclinic instabilities in Earth's oceans. J. Mar. Res., 65, 655-683, https://doi.org/ 10.1357/002224007783649484.

— , and J. Marshall, 2009: Evidence of enhanced mixing at middepth in the Southern Ocean. J. Phys. Oceanogr., 39, 50-69, https://doi.org/10.1175/2008JPO3880.1.

Stammer, D., 1998: On eddy characteristic, eddy transport and mean flow properties. J. Phys. Oceanogr., 28, 727-739, https://doi.org/10.1175/1520-0485(1998)028<0727:OECETA $>$ 2.0.CO;2.

Stone, P. H., 1966: On non-geostrophic baroclinic stability. J. Atmos. Sci., 23, 390-400, https://doi.org/10.1175/1520-0469 (1966) $023<0390$ :ONGBS $>2.0$. CO;2.

Tulloch, R., and Coauthors, 2014: Direct estimates of lateral eddy diffusivity upstream of Drake Passage. J. Phys. Oceanogr., 44, 2593-2616, https://doi.org/10.1175/JPO-D-13-0120.1.

Veneziani, M., A. Griffa, Z. Garrafo, and J. A. Mensa, 2014: Barrier layers in the tropical South Atlantic: Mean dynamics and sub-mesoscale effects. J. Phys. Oceanogr., 44, 265-288, https://doi.org/10.1175/JPO-D-13-064.1.

Visbeck, M. J., J. Marshall, J. Haine, and M. Spall, 1997: On the specification of eddy transfer coefficients in coarseresolution ocean circulation models. J. Phys. Oceanogr., 27, 381-402, https://doi.org/10.1175/1520-0485(1997)027<0381: SOETCI $>2.0 . C O ; 2$.

von Storch, J. S., C. Eden, I. Fast, H. Haak, D. Hernandez-Deckers, E. Maier-Reimer, J. Marotzke, and D. Stammer, 2012: An estimate of the Lorenz energy cycle for the world ocean based on a the $1 / 10^{\circ}$ STORM/NCEP simulation. J. Phys. Oceanogr., 42, 2185-2205, https://doi.org/10.1175/JPO-D-12-079.1.

Wilkin, J. L., and R. A. Morrow, 1994: Eddy kinetic energy and momentum flux in the Southern Ocean: Comparison of a global eddy-resolving model with altimeter, drifter and current meter data. J. Geophys. Res., 99, 7903-7916, https:// doi.org/10.1029/93JC03505.

WOCE, 2002: WOCE Global Data, version 3.0. WOCE Rep. 180/ 02, WOCE International Project Office, https://www.nodc. noaa.gov/woce/woce_v3/wocedata_1/diu/welcome.htm.

Wunsch, C., 1997: The vertical partition of oceanic horizontal kinetic energy. J. Phys. Oceanogr., 27, 1770-1794, https:// doi.org/10.1175/1520-0485(1997)027<1770:TVPOOH> 2.0. $\mathrm{CO} ; 2$. 